\title{
Demand-Based Optimal Design of Storage Tank with Inerter System
}

\author{
Shiming Zhang, Ruifu Zhang, and Zhipeng Zhao \\ Research Institute of Structural Engineering and Disaster Reduction, Tongji University, Shanghai 200092, China \\ Correspondence should be addressed to Ruifu Zhang; zhangruifu@tongji.edu.cn
}

Received 10 March 2017; Revised 20 June 2017; Accepted 12 July 2017; Published 20 September 2017

Academic Editor: Mario Terzo

Copyright (c) 2017 Shiming Zhang et al. This is an open access article distributed under the Creative Commons Attribution License, which permits unrestricted use, distribution, and reproduction in any medium, provided the original work is properly cited.

\begin{abstract}
A parameter optimal design method for a tank with an inerter system is proposed in this study based on the requirements of tank vibration control to improve the effectiveness and efficiency of vibration control. Moreover, a response indicator and a cost control indicator are selected based on the control targets for liquid storage tanks for simultaneously minimizing the dynamic response and controlling costs. These indicators are reformulated through a random vibration analysis under virtual excitation. The problem is then transformed from a multiobjective optimization problem to a single-objective nonlinear problem using the $\varepsilon$-constraint method, which is consistent with the demand-based method. White noise excitation can be used to design the tank with the inerter system under seismic excitation to simplify the calculation. Subsequently, a MATLAB-based calculation program is compiled, and several optimization cases are examined under different excitation conditions. The effectiveness of the demand-based method is proven through a time history analysis. The results show that specific vibration control requirements can be met at the lowest cost with a simultaneous reduction in base shears and overturning base moments.
\end{abstract}

\section{Introduction}

As a type of significant facility for public safety, liquid storage tanks play an important role in water supply systems, chemical industries, nuclear plants, and other fields. Storage tanks are designed in a wide range of capacity sizes ranging from small to large [1]. The vulnerability of tanks during earthquakes has been demonstrated in previous studies [26]. Based on the observations of several other researchers [710], the failure modes of tank damage can be divided into several types, such as buckling of tank walls, failure of link facilities, spillover of stored liquid, and uplifting of anchorage systems. Strengthening, isolation, and hybrid methods have been promoted by several researchers to ensure that storage tanks are not damaged during earthquakes [11-18].

The conventional technique of strengthening tanks to safeguard them against damage caused by earthquakes is to increase the size of different members to resist forces to a higher extent. Different from the strengthening methods, tanks are isolated from the period of the dominant component of ground motion, and the stiffness of the structures is adjusted using the isolation technique, which employs isolators between the base and the foundation of the tanks to increase their fundamental natural period. Shaking table tests have also been conducted to determine the effectiveness of the isolation method and demonstrate the reduction in the hydrodynamic pressure of these tanks and their seismic response $[19,20]$. However, this type of method can lead to an increase in the sloshing response $[8,21]$. The setting of obstacles, such as baffles, has been widely used to hinder liquid sloshing inside tanks and control structural damping. With baffles [22], the damping ratio of the sloshing mode increases while the liquid sloshing response decreases. The seismic energy is absorbed, and the resonant frequency of the sloshing mode increases because of the obstacles set in oscillating liquid. For the method involving baffles, there are some studies showing that baffles may cause an increase in the value of the base shear, and the ability of baffles in reducing sloshing effects in liquid storage tanks with lower aspect ratios is also under question [23]. Additionally, the provision for setting baffles is not available for some tanks with specific uses.

A hybrid control method that uses a viscous inerter system (VIS) and an isolator was proposed to control the 
earthquake response of storage tanks and simultaneously adjust the structure stiffness, damping ratio, and mass [21]. As a type of inerter system, the enhancement effect of mass and damping can be produced [8]. Therefore, the mass, damping ratio, and stiffness of the structure can be adjusted according to the structural requirement. The seismic response of tanks, such as their hydrodynamic pressure and sloshing response, can be efficiently reduced using the hybrid control method. A typical VIS is divided into two parts, namely, an inerter element and a damping element. The VIS used in this study is a type of parallel system, which consists of an inerter enhancement element and a viscosity element in parallel. The inerter element is a two-terminal mass element, which is different from the existing mass elements. Relative acceleration is allowed and constrained in the two-terminal inerter element model. The VIS can significantly dissipate seismic energy through the viscous element with the help of the inerter element. Luo et al. [21] proved the validity of the hybrid control method using theory and numerical calculation. The sloshing heights of the liquid, base shears, and overturning base moments of the tanks are decreased by applying the inerter system and an isolator. However, the methods of setting the design parameters of the inerter system, which achieves effective vibration control at the minimum cost under a specific controlling target, have not yet been proposed. The structural response of the tank is a complex problem under multiparameter settings, and structural control capability is continually adjusted as the parameters change.

Selecting the objective function of the design is critical in optimizing the design of the storage tank with the inerter system and determining the algorithm for calculating the function under multiple parameter changes. The fixed-point method proposed by Den Hartog [24] is applied to optimize the design of a tuned mass damper and can be extended for use in the VIS. Ikago et al. [8] adopted the fixed-point method for a tuned viscous mass damper. The disadvantage of the fixed method is that it neglects the inherent damping of the primary structure, and a limitation is introduced because an empirically equivalent mass ratio is used. Pan et al. [25] developed a demand-based optimal design for a single-degree-of-freedom structure considering the inherent damping ratio of the primary structure.

The dynamic response and force of the inerter system are reformulated according to the theory of random vibration to develop a suitable algorithm considering the storage tank's performance demand and synthetically controlling expense. Several variable parameters are changed in the investigation to optimize the selected objective indicators. Moreover, the optimization of the tank with the inerter system was converted to a multiobjective problem with the objective of synchronously minimizing the dynamic response and controlling cost. Considering two objective indicators, the $\varepsilon$ constraint approach is applied to convert the optimization problem to a demand-based (performance-based) design method with a demand level based on the limit values of the response of the sloshing height and the displacement of the isolation layer. A calculation program was compiled using the proposed demand-based design method to calculate the parameter optimization design. Finally, several optimization cases were considered under different excitation conditions, and a time history analysis was performed to exemplify the validity and accuracy of the proposed optimization method.

\section{Theoretical Analysis of the Storage Tank Controlled with the Inerter System}

2.1. Simplified Theoretical Model of the Storage Tank. Several theoretical models for analyzing the seismic response of storage tanks have been proposed to simplify the tank model for theoretical analysis and numerical calculation. Housner [27] proposed a type of lumped-mass model for theoretical analysis by analogizing the theoretical calculation model of shear force. The hypotheses were that the wall of the storage tank is rigid, the fluid displacements are small, fluid is incompressible, and the liquid in the storage tank can be divided into two components (i.e., convective and impulsive). Under the force of an earthquake, the impulsive component synchronously moves with the tank wall, whereas the convective component exhibits a sloshing motion. The researchers performed a shaking table experiment and proposed a more accurate consideration of higher-order vibration modes to verify the correctness of this model. Referring to a previously proposed model [28], a more accurate calculation model is selected in this study considering higher-sloshing modes to evaluate the seismic response of storage tanks.

Considering the first three sloshing mode components, the convective component in this model is divided into these three components. Its sloshing motion can be expressed as a linear combination of these modes. A rigid cylindrical tank is regarded as a four-lumped-mass model. Figure 1 shows the diagram of a tank controlled with the VIS and isolators. $D$ represents the diameter of the rigid tank, while $H$ represents the height of the liquid in the tank. $m_{j}, k_{j}, c_{j}$, and $h_{j}$ denote the mass, stiffness, damping coefficient, and height of the $j$ th sloshing mode, respectively. $\rho$ and $R$ denote the liquid density and the tank radius, respectively. $m_{j}, k_{j}, c_{j}$, and $h_{j}$ can be calculated by referring to the study of Luo et al. [21].

2.2. Simplified Model of Rubber Bearing. A rubber bearing (RB), which is set in the hybrid device, consists of a spring and a dashpot connected in parallel. The output force, $F$, provided by the RB can be expressed as follows:

$$
F=k_{b} x_{b}+c_{b} \dot{x}_{b}
$$

where $k_{b}$ denotes the stiffness of the spring, while $c_{b}$ represents the damping coefficient of the dashpot. $x_{b}$ and $\dot{x}_{b}$ denote the relative base displacement and relative base velocity, respectively.

2.3. Simplified Model of the VIS. The VIS used in this study is a type of inerter system comprising the following two parts: inerter element and viscous damping element.

Figure 2 shows the inerter enhancement element, which is a two-terminal element in which relative displacement is allowed and constrained. Different from the existing mass 


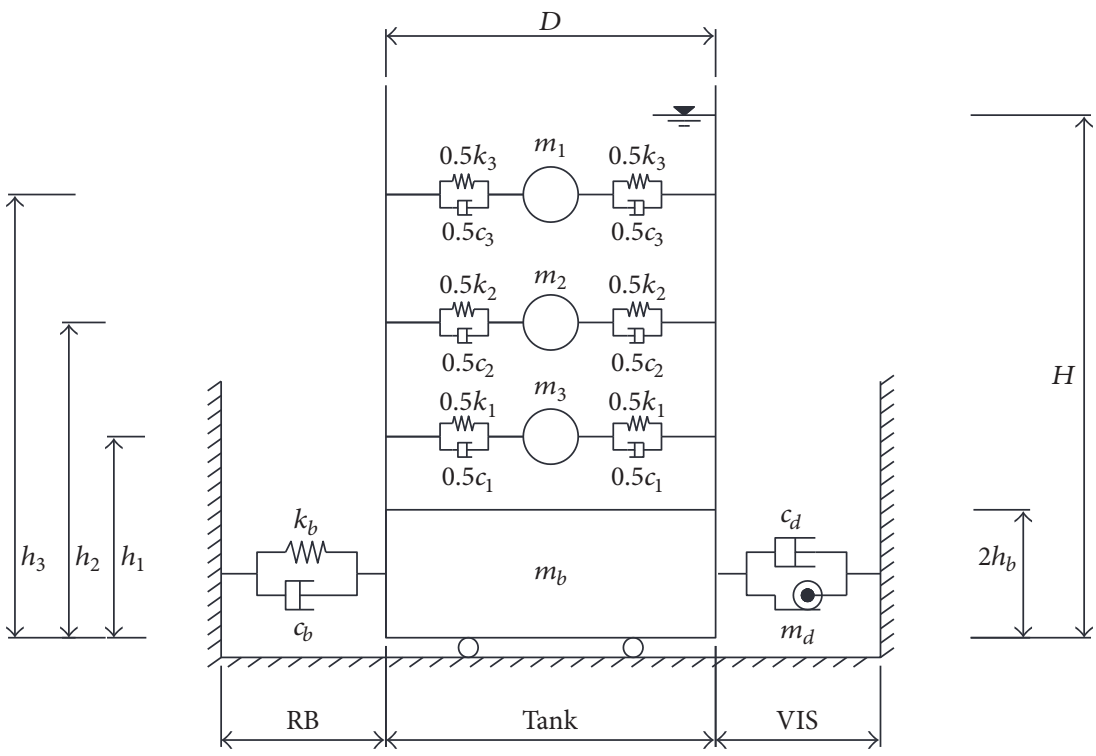

FIGURE 1: Simplified model of the controlled rigid tank with the inerter system.

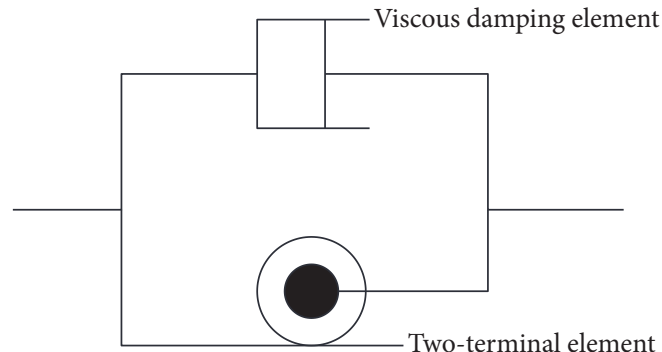

FIGURE 2: Schematic of the viscous damping element and the twoterminal inerter element.

model, the two-terminal element allows for relative acceleration within itself. More energy, which is efficiently dissipated by the viscous element, can be dissipated with the help of the amplified equivalent mass [8]. Therefore, according to the principle of Newtonian mechanics, the output force can be expressed as the vector product of the mass and the relative acceleration of the element.

As mentioned earlier, the inerter element is connected with the viscous damping element in parallel. The output force $F_{d}$ can be expressed using the following equation:

$$
F_{d}=c_{d} \dot{x}_{d}+m_{d} \ddot{x}_{d},
$$

where $c_{d}$ and $m_{d}$ denote the equivalent damping coefficient and the equivalent amplified mass of the VIS, respectively. $\dot{x}_{d}$ and $\ddot{x}_{d}$ denote the velocity and the acceleration of the VIS, respectively.

2.4. Analytical Model. The equation of motion for the tank controlled with the VIS and the isolators under the ground motion excitation, $\ddot{x}_{0}(\mathrm{t})$, can be written as follows:

$$
\left(\mathbf{M}_{\mathbf{s}}+\mathbf{M}_{\mathbf{a}}\right) \ddot{\mathbf{X}}+\left(\mathbf{C}_{\mathbf{s}}+\mathbf{C}_{\mathbf{a}}\right) \dot{\mathbf{X}}+\mathbf{K}_{\mathbf{s}} \mathbf{X}=-\mathbf{M}_{\mathbf{s}} \mathbf{I} \ddot{x}_{0}(t),
$$

where $\mathbf{M}_{\mathbf{s}}, \mathbf{K}_{\mathrm{s}}$, and $\mathbf{C}_{\mathbf{s}}$ are the mass, stiffness, and damping matrices of the tank, respectively; $\mathbf{M}_{\mathbf{a}}$ and $\mathbf{C}_{\mathbf{a}}$ are the additional mass and damping matrices provided by the VIS, respectively; $\mathbf{X}, \dot{\mathbf{X}}$, and $\dot{\mathbf{X}}$ are the vectors of displacement, velocity, and acceleration relative to the ground, respectively; and $\mathbf{I}$ is the influence coefficient vector. The matrices and vectors used in (3) are expressed as follows:

$$
\mathbf{M}_{\mathbf{s}}=\left(\begin{array}{cccc}
m_{b} & & & \\
& m_{1} & & \\
& & m_{2} & \\
& & & m_{3}
\end{array}\right) \text {, }
$$$$
\mathbf{C}_{\mathrm{s}}=\left(\begin{array}{cccc}
c_{b}+c_{1}+c_{2}+c_{3} & -c_{1} & -c_{2} & -c_{3} \\
-c_{1} & c_{1} & & \\
-c_{2} & & c_{2} & \\
-c_{3} & & & c_{3}
\end{array}\right) \text {, }
$$$$
\mathbf{K}_{\mathrm{s}}=\left(\begin{array}{cccc}
k_{b}+k_{1}+k_{2}+k_{3} & -k_{1} & -k_{2} & -k_{3} \\
-k_{1} & k_{1} & & \\
-k_{2} & & k_{2} & \\
-k_{3} & & & k_{3}
\end{array}\right) \text {, }
$$

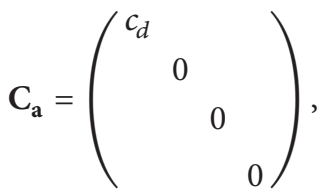

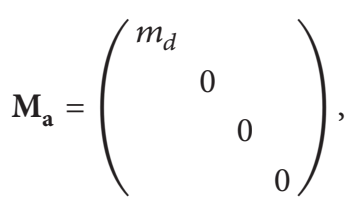




$$
\begin{gathered}
\mathbf{X}=\left\{\begin{array}{l}
x_{b} \\
x_{1} \\
x_{2} \\
x_{3}
\end{array}\right\}, \\
\dot{\mathbf{X}}=\left\{\begin{array}{l}
\dot{x}_{b} \\
\dot{x}_{1} \\
\dot{x}_{2} \\
\dot{x}_{3}
\end{array}\right\}, \\
\ddot{\mathbf{X}}=\left\{\begin{array}{l}
\ddot{x}_{b} \\
\ddot{x}_{1} \\
\ddot{x}_{2} \\
\ddot{x}_{3}
\end{array}\right\}, \\
\mathbf{I}=\left\{\begin{array}{l}
1 \\
1 \\
1 \\
1
\end{array}\right\} .
\end{gathered}
$$

A solution for the stochastic vibration response was obtained herein, based on the complex mode and pseudoexcitation method [29]. The equation of motion (i.e., (3)) is transformed into the equation of state of space as follows:

$$
\begin{aligned}
& \left(\begin{array}{cc}
\mathbf{O} & \mathbf{M}_{\mathrm{s}}+\mathbf{M}_{\mathrm{a}} \\
\mathbf{M}_{\mathrm{s}}+\mathbf{M}_{\mathrm{a}} & \mathbf{C}
\end{array}\right)\left(\begin{array}{l}
\ddot{\mathbf{X}} \\
\dot{\mathbf{X}}
\end{array}\right) \\
& \quad+\left(\begin{array}{cc}
-\left(\mathbf{M}_{\mathrm{s}}+\mathbf{M}_{\mathrm{a}}\right) & \mathbf{O} \\
\mathbf{O} & \mathbf{K}_{\mathrm{s}}
\end{array}\right)\left(\begin{array}{l}
\dot{\mathbf{X}} \\
\mathbf{X}
\end{array}\right)=\left(\begin{array}{c}
\mathbf{O} \\
-\mathbf{M}_{\mathbf{s}} \mathrm{I}
\end{array}\right) \ddot{x}_{0}(t) .
\end{aligned}
$$

The state space equation can be expressed as follows, considering the case of intense harmonic motion $\mathbf{X}=\varphi e^{\lambda t}$ :

$$
\mathbf{Y}=\left(\begin{array}{c}
\dot{\mathbf{X}} \\
\mathbf{X}
\end{array}\right)=\left(\begin{array}{c}
\lambda \varphi \\
\varphi
\end{array}\right) e^{\lambda t}=\varphi e^{\lambda t}
$$

Equation (5) can be expressed as follows to evaluate its eigenvalues and eigenvectors:

$$
\lambda\left(\begin{array}{cc}
\mathbf{O} & \mathbf{M}_{\mathrm{s}}+\mathrm{M}_{\mathrm{a}} \\
\mathbf{M}_{\mathrm{s}}+\mathrm{M}_{\mathrm{a}} & \mathrm{C}
\end{array}\right) \varphi=-\left(\begin{array}{cc}
-\left(\mathrm{M}_{\mathrm{s}}+\mathrm{M}_{\mathrm{a}}\right) & \mathbf{O} \\
\mathbf{O} & \mathrm{K}_{\mathrm{s}}
\end{array}\right) \varphi
$$

The eigenvalue matrix and the eigenvector matrix are obtained by solving (7) as follows:

$$
\begin{aligned}
& \boldsymbol{\lambda}=\left(\lambda_{1}, \lambda_{2}, \ldots, \lambda_{2 N}\right)^{T}, \\
& \boldsymbol{\Phi}=\left(\boldsymbol{\varphi}_{1}, \boldsymbol{\varphi}_{2}, \ldots, \boldsymbol{\varphi}_{2 N}\right),
\end{aligned}
$$

where $\lambda$ denotes the complex eigenvalue matrix and $\Phi$ denotes the complex eigenvector matrix.
Considering $\mathbf{Y}=\mathbf{\Phi Z}$ and substituting this into (5), the equation is diagonalized and expressed as follows:

$$
\dot{\mathbf{Z}}-\mathbf{\Lambda} \mathbf{Z}=\mathbf{R} \ddot{x}_{0}(t),
$$

where the parameters used in (9) are explained as follows:

$$
\begin{aligned}
\boldsymbol{\Lambda} & =\operatorname{diag}\left(\lambda_{1}, \lambda_{2}, \ldots, \lambda_{2 N}\right), \\
\mathbf{R} & =\operatorname{diag}\left(r_{1}, r_{2}, \ldots, r_{2 N}\right), \\
\ddot{x}_{0}(t) & =\sqrt{S_{g}(\omega)} \cdot e^{i \omega t},
\end{aligned}
$$

where $r_{m}=\boldsymbol{\varphi}_{m}^{T} \mathbf{F} / A_{m}, S_{g}(\omega)$ represents the ground input excitation power spectral density, and $\omega$ denotes the frequency of the external harmonic excitation. Differential equation (9) can be solved as follows:

$$
Z_{m}(\omega, t)=\frac{r_{m}}{i \omega-\lambda_{m}} \sqrt{S_{g}(\omega)} \cdot e^{i \omega t}=Z_{m}(\omega) \cdot e^{i \omega t} .
$$

According to the expression in (11), the response of the structure under virtual harmonic vibration excitation can be expressed as follows:

$$
\begin{aligned}
\mathbf{Y}(\omega, t) & =\sum_{m=1}^{2 N} \boldsymbol{\varphi}_{m} \cdot \frac{r_{m}}{i \omega-\lambda_{m}} \sqrt{S_{g}(\omega)} \cdot e^{i \omega t} \\
& =\mathbf{Y}(\omega) \cdot e^{i \omega t},
\end{aligned}
$$

where $N$ denotes the number of degrees of freedom.

The response of the power spectral density matrix can be expressed as follows:

$$
\begin{aligned}
& \mathbf{S}_{x x}(\omega)=\mathbf{X}^{*}(\omega) \mathbf{X}^{T}(\omega), \\
& \mathbf{S}_{\dot{x} \dot{x}}(\omega)=\dot{\mathbf{X}}^{*}(\omega) \dot{\mathbf{X}}^{T}(\omega), \\
& \mathbf{S}_{\ddot{x} \ddot{x}}(\omega)=\ddot{\mathbf{X}}^{*}(\omega) \ddot{\mathbf{X}}^{T}(\omega),
\end{aligned}
$$

where $\mathbf{X}^{*}, \dot{\mathbf{X}}^{*}$, and $\ddot{\mathbf{X}}^{*}$ denote the conjugates of the matrices of displacement, velocity, and acceleration relative to the ground, respectively. $\mathbf{X}^{T}, \dot{\mathbf{X}}^{T}$, and $\ddot{\mathbf{X}}^{T}$ denote the transposes of the matrices of displacement, velocity, and acceleration relative to the ground, respectively. The output force of the VIS power spectral density function can be determined as follows:

$$
\begin{aligned}
& S_{F d}(\omega) \\
& \quad=\left(m_{d} \ddot{X}_{b}^{*}(\omega)+c_{d} \dot{X}_{b}^{*}(\omega)\right)\left(m_{d} \ddot{X}_{b}^{T}(\omega)+c_{d} \dot{X}_{b}^{T}(\omega)\right) .
\end{aligned}
$$

\section{Optimization of the Storage Tank Controlled with the Inerter System}

3.1. Parametric Study of the Storage Tanks Controlled with the Inerter System. A parametric study was conducted to determine the responses by changing parameters $\mu_{c}, \mu_{m}$, and $s$ in the viable definition domain and investigate the reduction 
in the seismic response. The three parameters are defined as follows:

$$
\begin{aligned}
\mu_{m} & =\frac{m_{d}}{m_{b}}, \\
\mu_{c} & =\frac{c_{d}}{c_{b}}, \\
s & =\frac{2 H}{D},
\end{aligned}
$$

where $s$ is the aspect ratio of the tank (i.e., the ratio of the height of the tank to its radius); $\mu_{c}$ is the additional damping ratio, which is the ratio of the damping coefficients of the VIS and RB; and $\mu_{m}$ denotes the ratio of the equivalent amplified masses of the VIS and the impulsive components. Two dimensionless response variation ratios (i.e., the sloshing height and displacement of the isolation layer ratio, $\gamma_{s}$, and the output force ratio, $\gamma_{F d}$ ) were defined as follows to make the results of the study universally applicable:

$$
\begin{gathered}
\gamma_{s}\left(\mu_{c}, \mu_{m}, s\right)=\frac{\sigma_{s}}{\sigma_{s 0}}=\frac{\sqrt{\int_{-\infty}^{+\infty} S_{s s}(\omega) d \omega}}{\sqrt{\int_{-\infty}^{+\infty} S_{0 s s}(\omega) d \omega}}, \\
\gamma_{F d}\left(\mu_{c}, \mu_{m}, s\right)=\frac{\sigma_{F d}}{\sigma_{F d 0}}=\frac{\sqrt{\int_{-\infty}^{+\infty} S_{F d}(\omega) d \omega}}{\sqrt{\int_{-\infty}^{+\infty} S_{0 F d}(\omega) d \omega}} .
\end{gathered}
$$

$\gamma_{s}$ is the sloshing height and the displacement of the isolation layer ratio (i.e., the ratio of the sloshing height and the displacement of the tank with the inerter system and the uncontrolled tank) of the tank. $\gamma_{F d}$ is the output force ratio, which is the ratio of the output force of the tank to the inerter system and the uncontrolled tank (caused by inherent damping). $\sigma_{F d 0}$ and $\sigma_{s 0}$ are the root mean square (RMS) values of the output force and the sloshing height responses, respectively, combined with the displacement of the isolation layer of the uncontrolled tank. $\sigma_{F d}$ and $\sigma_{s}$ are the RMS values of the output force and the sloshing height responses, respectively, combined with the displacement of the isolation layer of the tank controlled with the VIS.

Considering the control of the sloshing height of the liquid in the tank, the sloshing height response, $S$, refers to the sum of the sloshing heights of the first three modes of liquid vibration. As mentioned above, the sloshing motion of the convective component can be expressed as a linear superposition of the natural modes of the liquid vibration. The sloshing height, $S$, can then be determined using the following equation:

$$
S(t)=\sum_{j=1}^{3} S_{j}(t)=\frac{R}{g} \sum_{j=1}^{3} \frac{2}{\lambda_{j}^{2}-1}\left(\ddot{x}_{j}(t)+\ddot{x}_{0}(t)\right),
$$

where $S_{j}$ denotes the sloshing height in the $j$ th mode; $\lambda_{j}$ denotes the values for which the first derivative of the Bessel function of the first kind and first order is zero; and $\lambda_{1}$, $\lambda_{2}$, and $\lambda_{3}$ are $1.841,5.331$, and 8.536, respectively. Therefore, $S_{s s}(\omega)$ in (16) can be determined as follows:

$$
\begin{aligned}
& S_{s s}(\omega) \\
& =\frac{R}{g} \sum_{j=1}^{3} \frac{2}{\lambda_{j}^{2}-1}\left(S_{\ddot{x} \ddot{x} j}(\omega)+S_{\ddot{x} \ddot{x} 0}(\omega)\right)+0.2 \times S_{x x b}(\omega),
\end{aligned}
$$

where $S_{\ddot{x} \ddot{x} j}(\omega)$ and $S_{\ddot{x} \ddot{x} 0}(\omega)$ denote the power spectral density of the acceleration response and the ground motion of the $j$ th mode, respectively. $S_{x x b}(\omega)$ is the displacement of the power spectral density of the impulsive components. Substituting (14) into (16) and (19), these two objective indicators can be accurately calculated. A toolbox for integration in MATLAB is used for overcoming the considerable difficulties in performing the integration in (16) and (17). Indicators $\gamma_{s}$ and $\gamma_{F d}$ can be obtained as the sum of the corresponding index values at different time points by adopting the iterative calculation method and defining the numerical calculation steps.

A three-dimensional (3D) space can be established to demonstrate the changes in the indicator values and enhance the visual representation of the calculation results considering the three undetermined parameters, $\mu_{c}, \mu_{m}$, and $s$. The parameters in the $3 \mathrm{D}$ space are set as the orthogonal coordinate axes. Each point in the parametric space can be assigned a specified color based on the corresponding value of the response of the controlled tank to clearly visualize the results of the responses. Slices can be made at specific parametric planes to investigate the variation in the response with respect to the changes in the undetermined parameters $\left(\mu_{c}, \mu_{m}\right.$, and $\left.s\right)$. The target benchmark is set as the storage tank for which the values of $\mu_{c}, \mu_{m}$, and $s$ are $0.5,0.5$, and 1.5 , respectively.

A set of array-specific slices at constant $\mu_{c}, \mu_{m}$, and $s$ are used in the $3 \mathrm{D}$ contour map to observe and obtain an intuitive prediction of the variation in the objective function over the three parameters. The corresponding trends of indicators $\gamma_{s}$ and $\gamma_{F d}$ that changed with the three parameters are also compared. Figure 3 shows the following adopted parametric ranges:

$$
\begin{aligned}
0.01 & <\mu_{c}<1.0, \\
0.01 & <\mu_{m}<1.0, \\
0.5 & <s<3.0 .
\end{aligned}
$$

The ratio of the sloshing height to the displacement of the isolation layer, $\gamma_{s}$, of the viscous liquid was selected as the objective indicator in the $3 \mathrm{D}$ space considering the seismic response of the tank controlled using the hybrid method and the liquid stability. The slices shown in Figure 3 denote that only two parameters can be varied in each contour plot. Obtaining the influence of parameter variation on the seismic response of the controlled tank is possible by analyzing the change in the objective indicator for different slices.

The analysis of the global contour color implies that the response of the sloshing height of the liquid in the tank varies with the three variables. The minimum value of $\gamma_{s}$ is observed at a fixed point or a boundary of the contour plot, denoting 


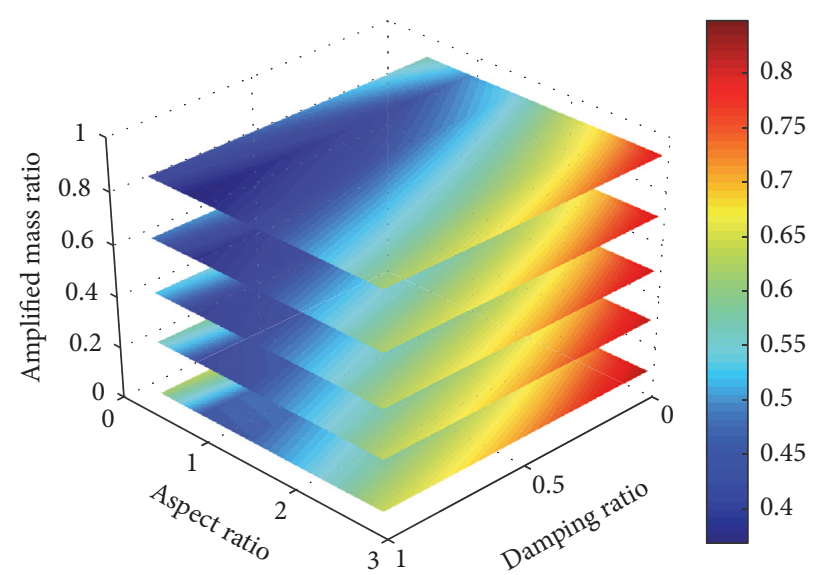

(a) Slices at the constant amplified mass ratio values $\left(\mu_{m}=0.1,0.3,0.5\right.$, $0.7,0.9)$

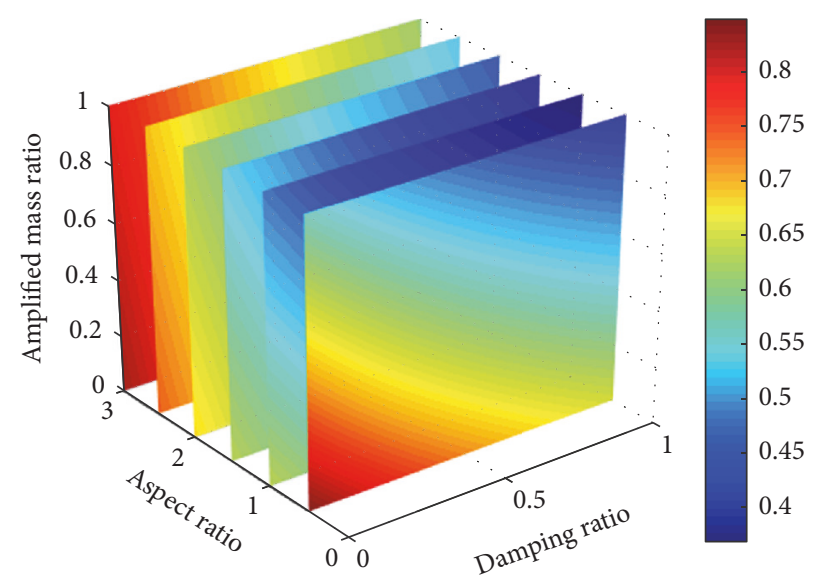

(b) Slices at the constant aspect ratio values $(s=0.5,1.0,1.5,2.0,2.5,3.0)$

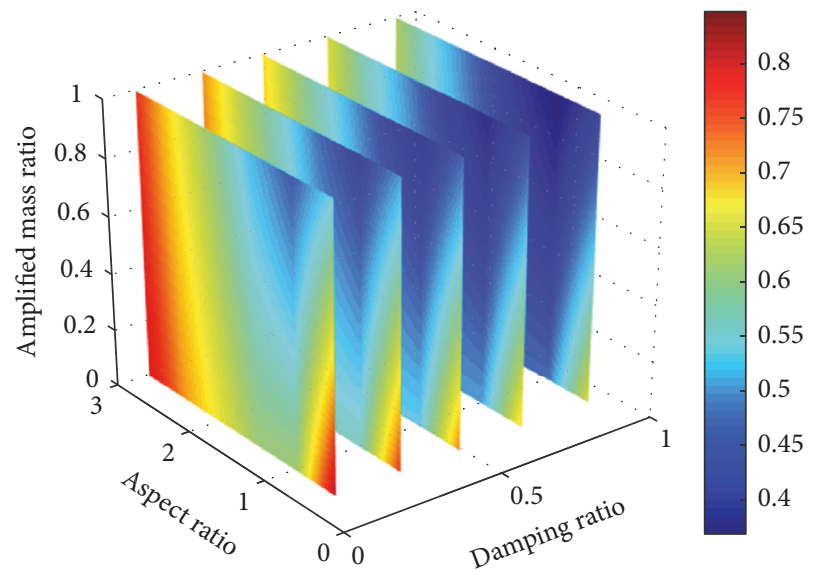

(c) Slices at the constant damping ratio values $\left(\mu_{c}=0.1,0.3,0.5,0.7,0.9\right)$

FIGURE 3: Sloshing height ratio $\gamma_{s}$ in the $\mu_{c}, \mu_{m}, s$ space of the storage tank with the VIS.

that the structural control objectives will be achieved under the conditions of maximum output force and mass force if the height-to-diameter ratio of the tank, $s$, damping ratio, $\mu_{c}$, and mass parameters of the tank are adjusted freely during the seismic tank design. In actual design, the tank geometry is always preset based on specific requirements and manufacturing capacity. Therefore, the optimal design based on the fixed aspect ratio is more valuable in practical view.

(1) To analyze the slices at several selected aspect ratios, it can be considered that, under the conditions of a specific height-to-diameter ratio, there is no closed loop in the variation of parameters $\mu_{c}$ and $\mu_{m}$. This implies that, without a preset parameter variation range, the indicator index of the controlled tank will be minimized when the damping ratio and the amplified mass ratio reach their maximum values. The minimum value of $\gamma_{s}$ described above will be at the expense of high control cost of the VIS.

(2) It can be clearly observed in the slices at several selected damping ratios that, for a specific damping ratio, the value of indicator $\gamma_{s}$ changes with the two parameters. As the aspect ratio of the tank decreases, the tank with a low aspect ratio exhibits a better controlling effect of the sloshing height and the displacement of the isolation layer under the same damping ratio. The comparison of different slices shows that the variation in $\mu_{m}$ has a negligible effect on the numerical value of the control target. Therefore, the efficiency of optimizing $\mu_{m}$ is not satisfactory under the condition of a preset specific damping ratio. As shown in these slices, the optimization of the tank geometry is extremely efficient and operable.

(3) The same trend as that for the slices at several selected damping ratios is observed in the analysis of the slices at several selected amplified mass ratios. A comparison of the slices at different amplified ratios shows that the value of $\gamma_{s}$ is extremely sensitive to the changes in the aspect ratio. The value of $\gamma_{s}$ for tanks with a low aspect ratio does not significantly change with the damping ratio. Conversely, the value becomes more sensitive to the changes in the damping ratio as the aspect ratio of the tank increases. 
3.2. Optimization of the Hybrid Method Design. Preliminary conclusions on the control optimization can be obtained in the analysis of the 3D contour plot through a dynamic analysis of the controlled tank. According to the actual production capacity and device design requirements, the fluctuation in the sloshing height of the liquid in the storage tank will reach the minimum value at a high control cost within the range of the three variable parameters $\left(\mu_{c}, \mu_{m}\right.$, and $s$ ). In the actual optimization design of the VIS, certain control requirements are necessary to lower the control costs [25]. As mentioned above, $\gamma_{s}$ and $\gamma_{F d}$ are selected as the objective indicators. $\gamma_{F d}$ reflects the cost of structural control to a certain extent.

Furthermore, the target function for the optimization of the tank controlled with the VIS and isolations can be expressed as follows, considering the two indicators:

$$
\begin{array}{ll}
\operatorname{minimize} & {\left[F_{s}(x), F_{F d}(x)\right]} \\
\text { subject to } & x \in X
\end{array}
$$

where $F_{s}(x)$ and $F_{F d}(x)$ denote the target functions for the sloshing height and the output force of the VIS. $x$ denotes the vector of the three selected parameters. $X$ is the range of the definition field. $F_{s}(x)$ and $F_{F d}(x)$ are determined by selecting $\gamma_{s}$ and $\gamma_{F d}$, respectively. The target function (21) can be rewritten as follows, based on the optimization objectives:

$$
\begin{array}{ll}
\operatorname{minimize} & {\left[\gamma_{s}\left(\mu_{c}, \mu_{m}, s\right), \gamma_{F d}\left(\mu_{c}, \mu_{m}, s\right)\right]} \\
\text { subject to } & \left(\mu_{c}, \mu_{m}, s\right) \in X \\
& X=\left\{\begin{array}{l}
\mu_{c \min }<\mu_{c}<\mu_{c \max } \\
\mu_{m \min }<\mu_{m}<\mu_{m \max } \\
s_{\text {min }}<s<s_{\text {max }} .
\end{array}\right.
\end{array}
$$

In function (22), $\mu_{m \min }, \mu_{c \min }$, and $s_{\min }$ represent the lower bounds of the ranges of values, while $\mu_{\text {mmax }}, \mu_{c \max }$, and $s_{\max }$ represent the upper bounds of the ranges of values.

The $\varepsilon$-constraint method of the control theory is adopted to determine the optimal solution of the objective function. As discussed earlier, this optimization problem is a multiobjective problem (MOP) with two optimization goals. A few simplifications are made according to the control theory to solve this problem effectively. An objective function of the MOP is selected as the objective function of the SOP to transform the problem into an equivalent optimization problem with a single-objective function (i.e., a singleobjective problem (SOP)). The other objective function is treated as an extra constraint condition for the SOP by setting bound values. Similarly, in performance-based structural design theory, the performance levels are always expressed as response limit values. Target function (22) can be modified and expressed as follows using this method:

$$
\begin{array}{cl}
\operatorname{minimize} & \gamma_{F d}\left(\mu_{c}, \mu_{m}, s\right) \\
\text { subject to } & \gamma_{s}\left(\mu_{c}, \mu_{m}, s\right)<\gamma_{s . l i m} \\
& \mu_{c \min }<\mu_{c}<\mu_{c \max } \\
& \mu_{\min }<\mu_{m}<\mu_{\operatorname{mmax}} \\
& s_{\min }<s<s_{\max },
\end{array}
$$

where $\gamma_{s . l i m}$ denotes the upper limit of $\gamma_{s}$, which can be determined by design or functional requirements.

During the process of controlling the tank, a few supplementary constraints are required to be set to increase the controlling force provided by the inerter enhancement element and ensure that the inerter enhancement element of the VIS is in a dominant position in the seismic response during control. In other words, this constraint condition ensures that the damper is a type of inerter system that will not degenerate into a viscous damper. The constraint condition can be determined as follows based on this discussion:

$$
\psi_{F} \cdot \sigma_{F d, c}\left(\mu_{c}, \mu_{m}, s\right) \leq \sigma_{F d, m}\left(\mu_{c}, \mu_{m}, s\right) \quad 1 \leq \psi_{F} \leq 3 .
$$

Using this constraint condition, the inequality for factor $\psi_{F}$ ensures that the horizontal force provided by the rotating mass element dominates the VIS. In addition, this constraint satisfies the requirements of the type of inerter system used in the actual design optimization of the damping device.

A numerical calculation is conducted to confirm the existence of a unique optimization result. Based on the requirement of performance and its geometry design, the hypothesis in this benchmark model is that the aspect ratio is 1.5. For variation in only two parameters, the problem can be demonstrated in a plane (Figure 4). A series of response control demands and the constraint optimization problem are clearly demonstrated in the mixed contour plots of this plane. In addition, in each control demand, the lowest point on the contour of the damping ratio along any solid line represents a unique optimization result. Figure 4 shows a color-filled contour that represents the values of $\gamma_{F d}$ and solid lines in the plane that represent the values of $\gamma_{s}$. For every solid line, a unique lowest point in the color-filled contour of the output force represents a unique solution to the constraint optimization problem. In addition, the contour plot shows that the lower level of $\mu_{c}$ has less influence on the control target, and the mass coefficient is more sensitive to this variation. This finding illustrates the effectiveness of larger masses for sloshing height control.

The target benchmark is selected as the storage tank with $\mu_{c}, \mu_{m}$, and $s$ as $0.5,0.5$, and 1.5 , respectively, to clearly illustrate the control principle of the VIS. Figure 5 shows the response density curves of the tank with the VIS and an uncontrolled tank under simple harmonic vibration excitation. The figure illustrates that peaks are observed in the output force of the VIS and the dynamic response of the sloshing height when the external excitation frequency is close to that of the tank. With the VIS attached to the tank, 


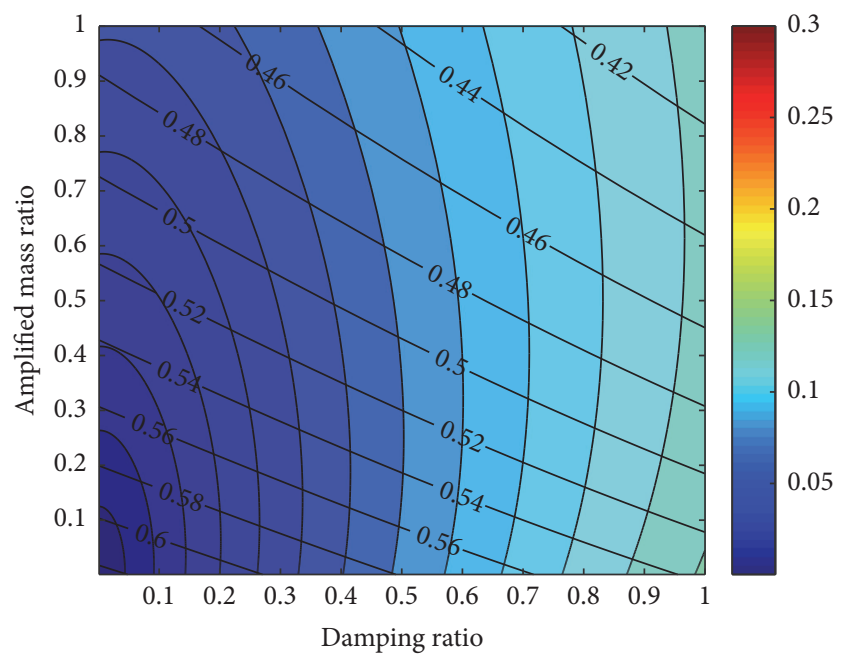

(a) Combined contour plot of $\gamma_{s}$ and $\gamma_{F d}$ at $s=1.5$

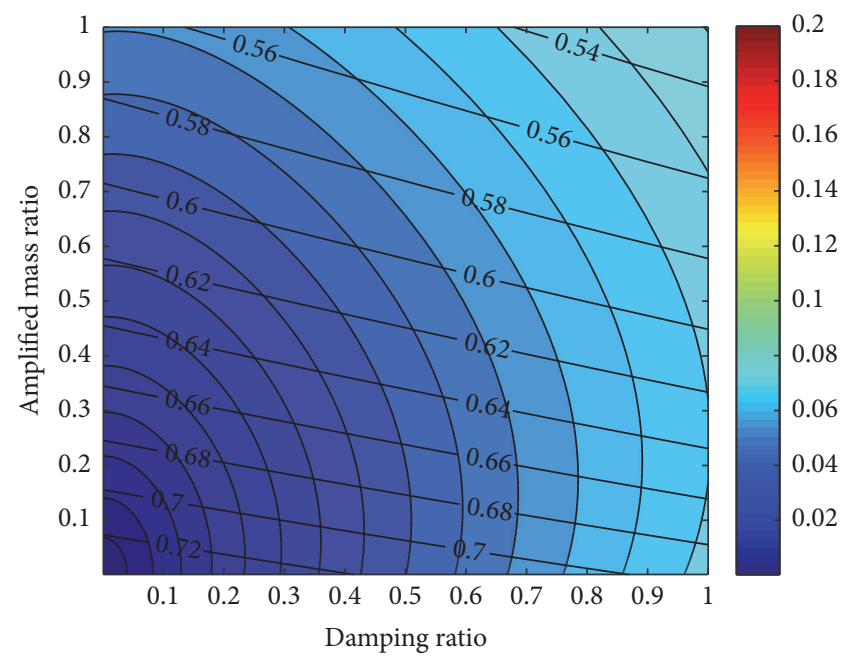

(b) Combined contour plot of $\gamma_{s}$ and $\gamma_{F d}$ at $s=2.5$

FIGURE 4: Combined contour plots of $\gamma_{s}$ and $\gamma_{F d}$ (the color-filled contour represents the values of $\gamma_{F d}$, while the solid lines in the plane represent the values of $\gamma_{s}$ ).

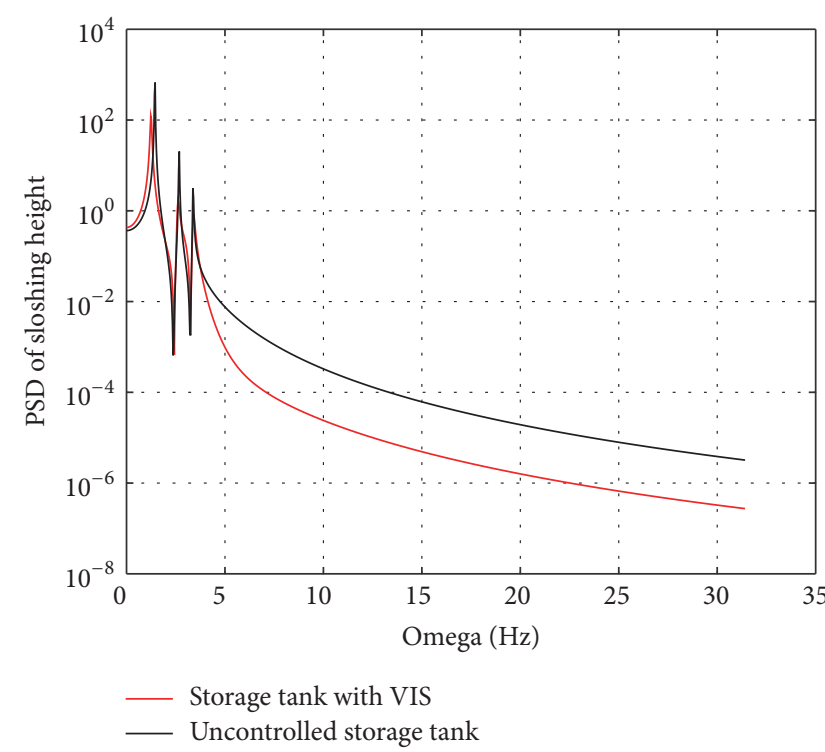

(a) Power spectral density of the sloshing height of the tank with the VIS and the uncontrolled tank

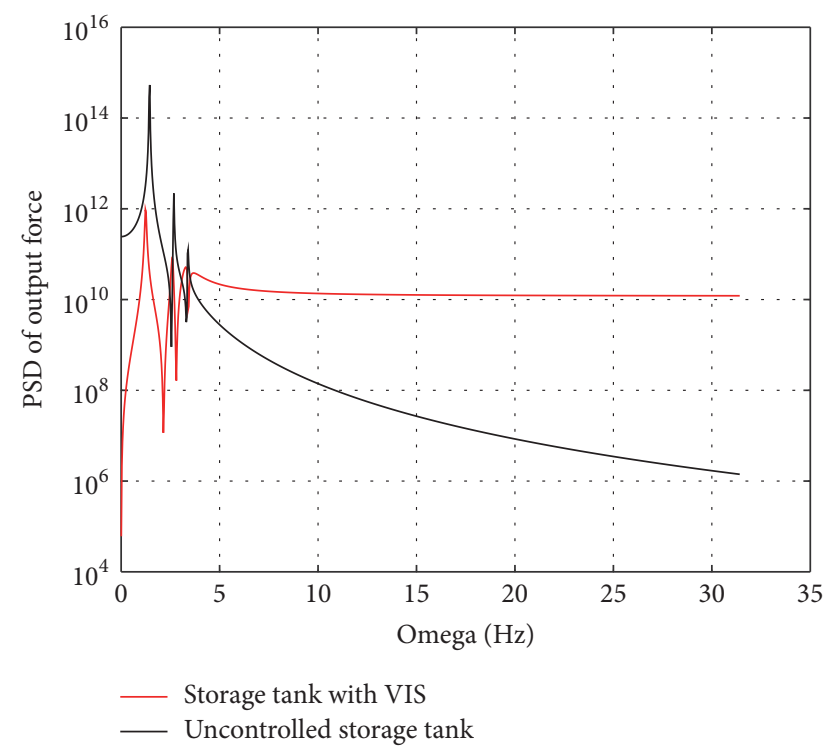

(b) PSD of output force of the tank with the VIS and the uncontrolled tank

FIGURE 5: Power spectral density (PSD) curves of the tank with VIS $\left(\mu_{c}=0.5, \mu_{m}=0.5\right.$, and $\left.s=1.5\right)$ and the uncontrolled tank.

the effect of sloshing height control is significantly enhanced, accompanied by a substantial increase in the output force, which implies lower control targets at the expense of higher control costs. In other words, the objective of the optimal design of the tank is to find the balance of the control target and the control cost.

3.3. Analysis of the Base Shears and the Overturning Base Moments. The base shears and the overturning base moments are considered and calculated according to the following expressions to complete the analysis of the dynamic response of the tank:

$$
\begin{gathered}
F_{b}=m_{b}\left(\ddot{x}_{b}+\ddot{x}_{0}\right)+\sum_{j=1}^{3} m_{j}\left(\ddot{x}_{j}+\ddot{x}_{0}\right), \\
M_{b}=m_{b} h_{b}\left(\ddot{x}_{b}+\ddot{x}_{0}\right)+\sum_{j=1}^{3} m_{j} h_{j}\left(\ddot{x}_{j}+\ddot{x}_{0}\right) .
\end{gathered}
$$




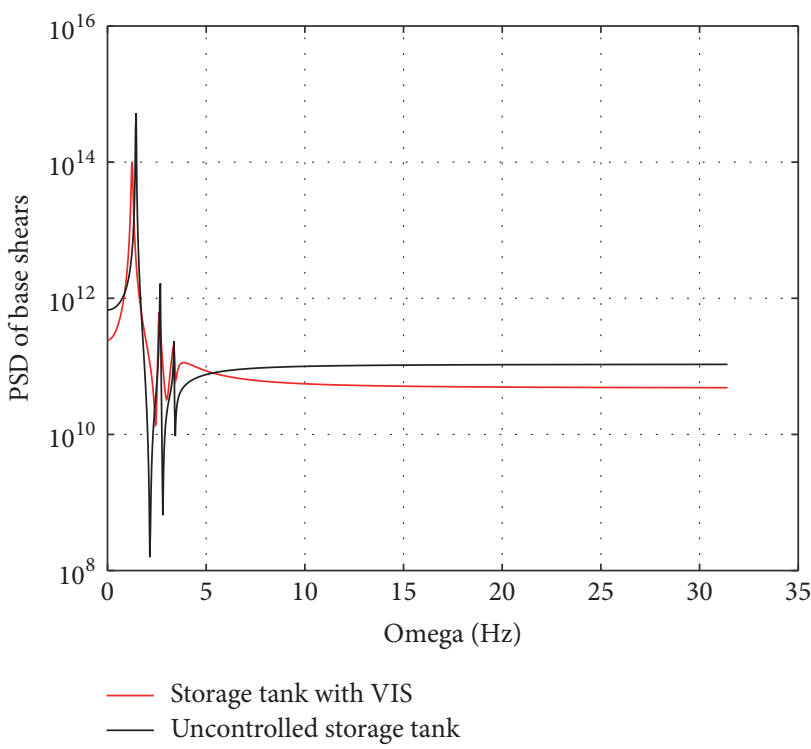

(a) PSD of the base shears of the tank with the VIS and the uncontrolled tank

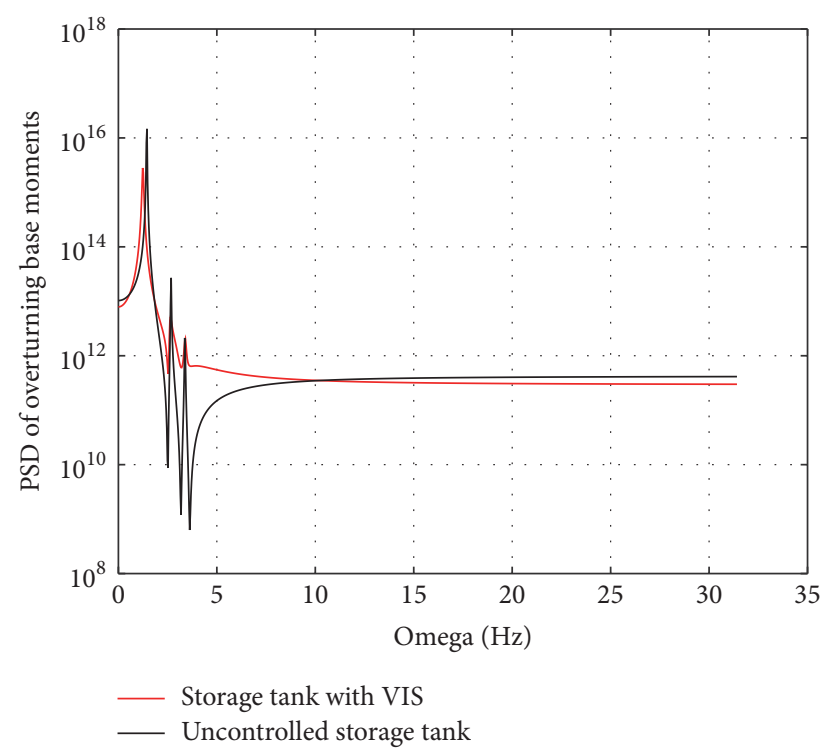

(b) PSD of the overturning base moments of the tank with the VIS and the uncontrolled tank

Figure 6: Power spectral density (PSD) curves of the benchmark tank $\left(\mu_{c}=0.5, \mu_{m}=0.5\right.$, and $\left.s=1.5\right)$ and the uncontrolled tank.
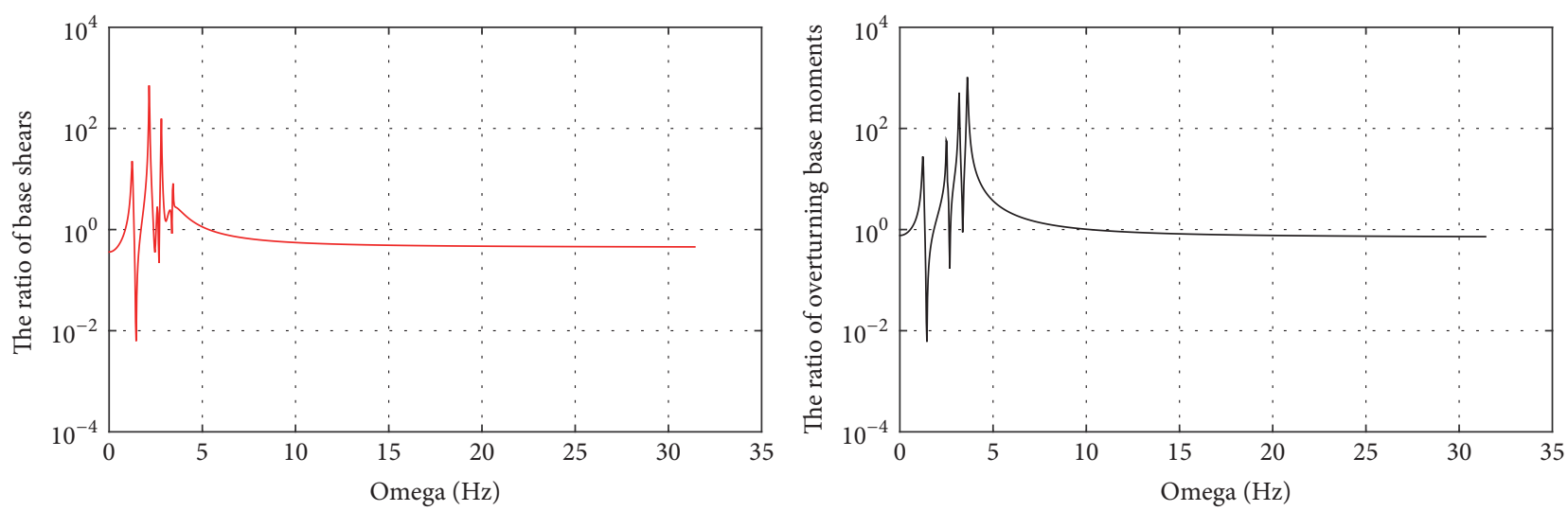

FIGURE 7: Curves of the ratios of the base shears and the overturning base moments.

The power spectral density of the base shears and the overturning base moments can be expressed as follows, based on (25):

$$
\begin{gathered}
S_{F_{d}}=\left(m_{b}\left(\ddot{X}_{b}^{*}+\ddot{X}_{0}^{*}\right)+\sum_{j=1}^{3} m_{j}\left(\ddot{X}_{j}^{*}+\ddot{X}_{0}^{*}\right)\right) \\
\cdot\left(m_{b}\left(\ddot{X}_{b}^{T}+\ddot{X}_{0}^{T}\right)+\sum_{j=1}^{3} m_{j}\left(\ddot{X}_{j}^{T}+\ddot{X}_{0}^{T}\right)\right), \\
S_{M_{b}}=\left(m_{b} h_{b}\left(\ddot{X}_{b}^{*}+\ddot{X}_{0}^{*}\right)+\sum_{j=1}^{3} m_{j} h_{j}\left(\ddot{X}_{j}^{*}+\ddot{X}_{0}^{*}\right)\right) \\
\cdot\left(m_{b} h_{b}\left(\ddot{X}_{b}^{T}+\ddot{X}_{0}^{T}\right)+\sum_{j=1}^{3} m_{j} h_{j}\left(\ddot{X}_{j}^{T}+\ddot{X}_{0}^{T}\right)\right) .
\end{gathered}
$$

The power spectral density of the overturning base moments and the base shears in the standard model can be calculated using (26). Figure 6 shows the results.

$\gamma_{F b}$ and $\gamma_{M b}$ are defined as the ratios of the base shears and the overturning base moments, respectively, to simplify the curves of the power spectral density and intuitively compare the benchmark and uncontrolled tanks. Figure 7 depicts the curves of $\gamma_{F b}$ and $\gamma_{M b}$, implying that the base shears and the overturning base moments of the tank with the VIS simultaneously decrease during the shortperiod external excitation and increase during the longperiod excitation. With the increase in the external excitation period, the dominant period can be close to the first period of the sloshing mode, which is an essential and larger modal participation factor of the base shears and the overturning base moments. 


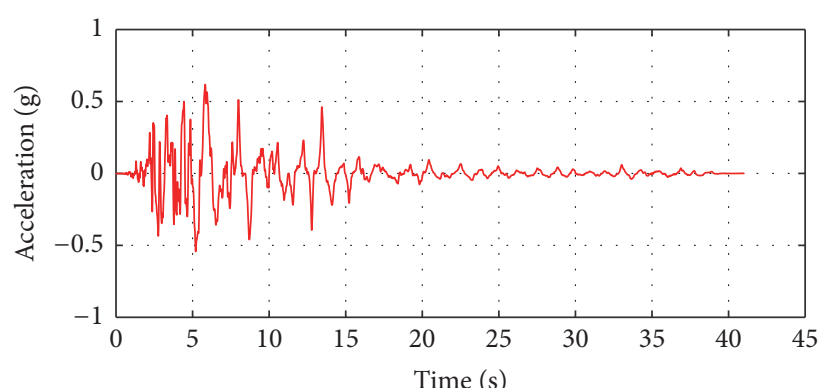

— Kobe-NS

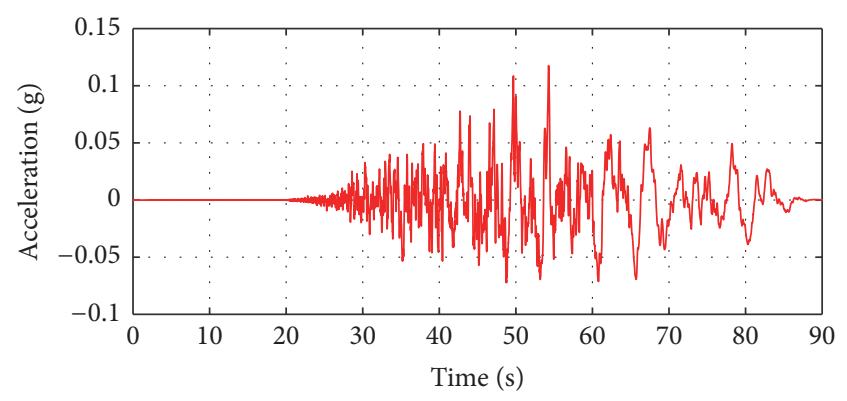

— Chi-Chi-NS

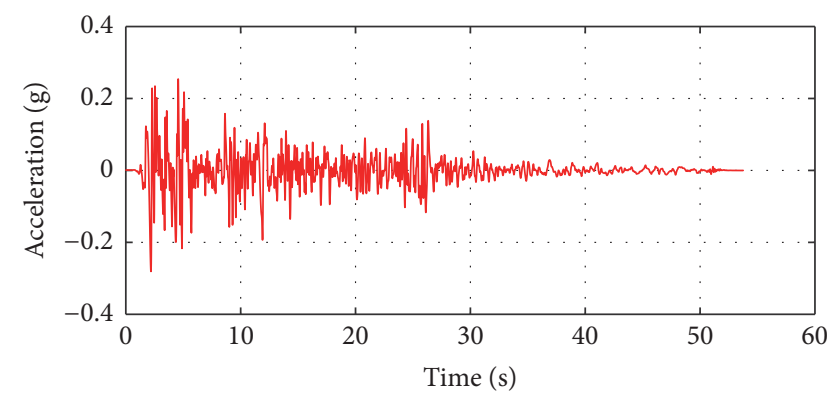

- El Centro-NS

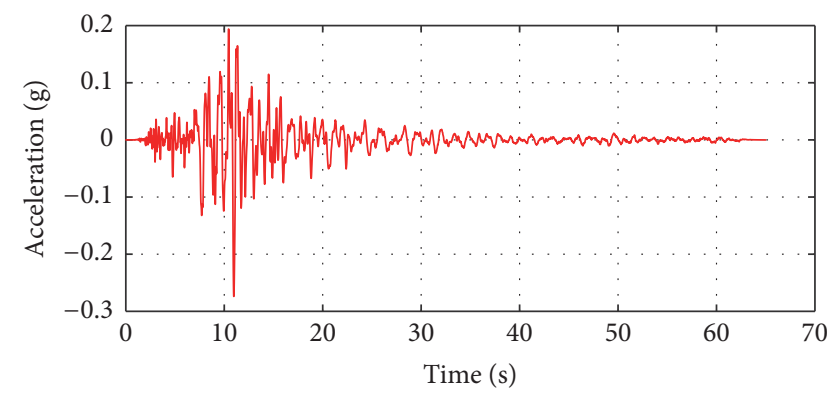

— Coalinga-EW

FIGURE 8: Time histories of the ground motions considered in this study.

\section{Design Example}

Based on the abovementioned discussion and the establishment of the tank calculation model under the control of the VIS, MATLAB is adopted to write the optimization program for the demand-based calculation. In the optimization process, the target index is optimized using the nonlinear constrained optimization algorithm in a MATLAB toolbox. This algorithm is compiled to obtain a constrained minimum of a scalar function of several variables starting from an initial estimate. This is typically referred to as the constrained nonlinear optimization or nonlinear programming.

Based on the discussion of Luo et al. [21], the tank controlled using the hybrid method can be effectively controlled during earthquakes. The actual tank vibration control is frequently based on the preset tank geometry. Therefore, the tank with an aspect ratio of 1.5 is selected as the benchmark. Table 1 lists the design conditions of the tanks at different sloshing height ratios. Parameter $\psi_{F}$, which is set as 1.5 , is included in the optimization calculation to ensure the dominance of the mass block in the VIS.

Table 2 lists the optimization results of each parameter and illustrates that the parameter optimization design under white noise excitation is similar to the other types of noises. In addition, it shows that white noise can be used to simplify the optimization design.

A series of time history dynamic analyses were conducted based on the optimization results for group $\mathrm{WN}$ 1 to verify the controlling effect of the optimally designed VIS-controlled tank. The target-based baseline correction method can correct the inconsistent vibration acceleration signals [30, 31]. Figure 8 shows the selected earthquake
TABLE 1: Design cases for the hybrid-controlled tank.

\begin{tabular}{lcc}
\hline Case ID & $s$ & $\gamma_{s}$ \\
\hline WN-1 & 1.5 & $45 \%$ \\
WN-2 & 1.5 & $50 \%$ \\
WN-3 & 1.5 & $55 \%$ \\
WN-4 & 1.5 & $60 \%$ \\
SS-1 & 1.5 & $45 \%$ \\
SS-2 & 1.5 & $50 \%$ \\
SS-3 & 1.5 & $55 \%$ \\
SS-4 & 1.5 & $60 \%$ \\
\hline
\end{tabular}

Kanai-Tajimi's spectrum [26] was adopted as the seismic input power spectrum. "Severest seismic (SS) excitation" implies that the predominant frequency of seismic excitation is equal to the first natural frequency.

waves. Figures 9-12 depict the representative time history response curves, which illustrate that the sloshing height response of every tank is effectively controlled by the VIS. Figure 13 presents the corresponding maximum sloshing heights. Meanwhile, Figures 14-17 illustrate that the base shears and the overturning base moments of the tank with the VIS are effectively reduced under the excitation of the El Centro, Kobe, and Coalinga earthquakes. In the case of the Chi-Chi earthquake, the base shears and the overturning base moments are observed to increase in the long-period earthquake. Figures 18-21 illustrate that the output force is higher in the early stage of earthquake excitation and decreases as the earthquake continues. Through parameter optimization, the output force can be effectively reduced under the premise of setting the vibration control target. In 
TABLE 2: Optimization results for the parameters of the hybrid-controlled tank.

\begin{tabular}{lcccc}
\hline \multirow{2}{*}{ Case ID } & \multicolumn{2}{c}{ Optimized design parameters } & Appendant damping force ratio & \multicolumn{2}{c}{ Sloshing height ratio } \\
& $\mu_{c}$ & $\mu_{m}$ & $\gamma_{d}$ & $4.62 \%$ \\
WN-1 & 1.0000 & 0.3112 & $4.67 \%$ & $50 \%$ \\
WN-2 & 0.6961 & 0.0484 & $2.64 \%$ & $55 \%$ \\
WN-3 & 0.3560 & 0.0204 & $0.83 \%$ & $60 \%$ \\
WN-4 & 0.1018 & 0.0048 & $6.10 \%$ & $45 \%$ \\
SS-1 & 0.9724 & 0.3478 & $4.14 \%$ & $50 \%$ \\
SS-2 & 0.6119 & 0.1862 & $2.37 \%$ & $55 \%$ \\
SS-3 & 0.3249 & 0.0853 & $0.75 \%$ & $60 \%$ \\
SS-4 & 0.0953 & 0.0218 & & \\
\hline
\end{tabular}

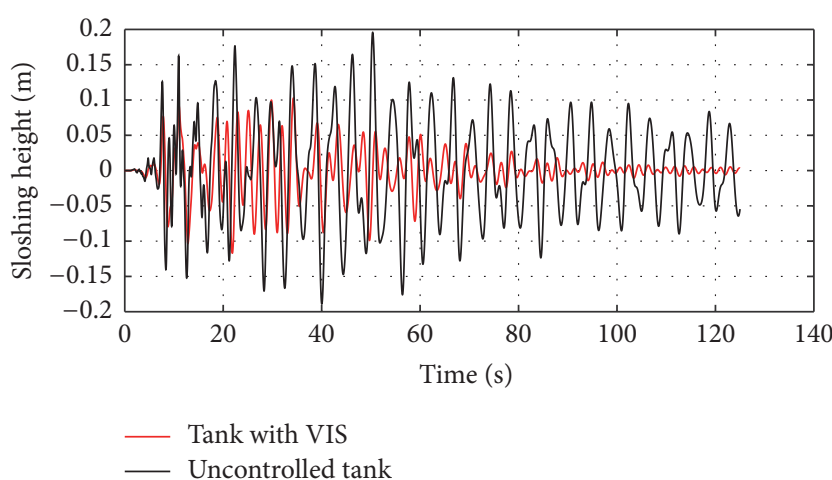

FIGURE 9: Dynamic responses of the sloshing height of the tank with the VIS under the excitation of the Coalinga earthquake.

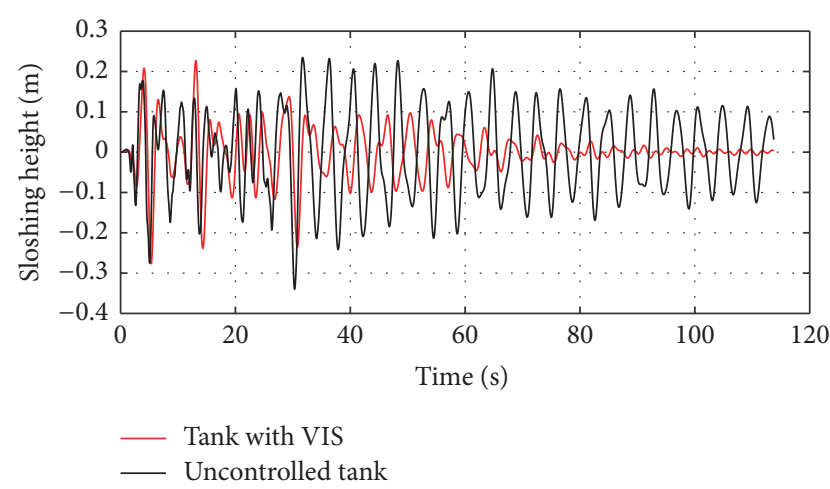

FIgURE 10: Dynamic responses of the sloshing height of the tank with the VIS under the excitation of the El Centro earthquake.

addition, the dynamic analysis results confirm the validity and the efficiency of the demand-based design optimization method of tank control.

\section{Conclusions}

This study proposes an optimal design method based on the tank vibration performance and applies this to the optimization of tank control with an inerter system. This design

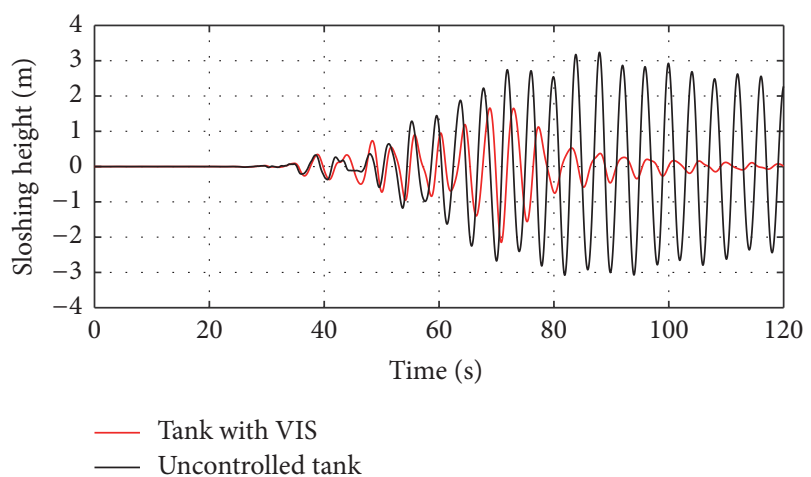

FIGURE 11: Dynamic responses of the sloshing height of the tank with the VIS under the excitation of the Chi-Chi earthquake.

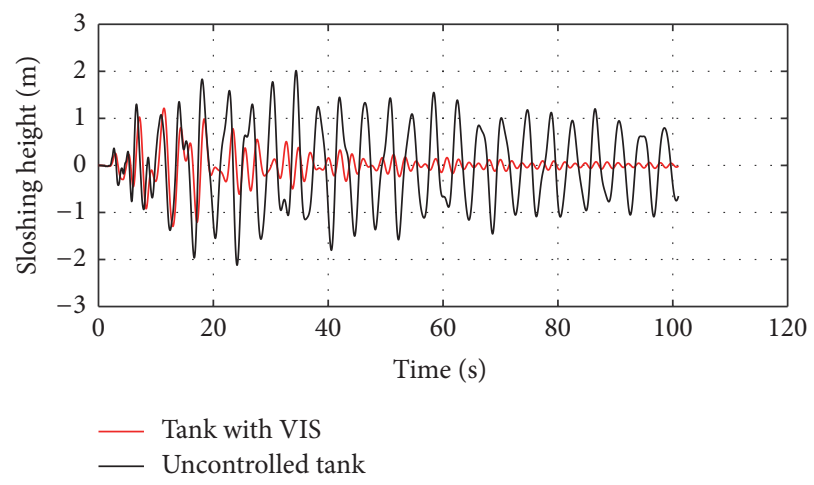

FIGURE 12: Dynamic responses of the sloshing height of the tank with the VIS under the excitation of the Kobe earthquake.

method provides an effective solution for the parameter optimization design of the VIS and overcomes the problems in existing design methods. Based on the theory of stochastic vibration, the optimization program is compiled based on selected optimization indicators. The effectiveness of the method is also proven through a time history analysis. The following conclusions can be drawn from the study:

(1) The optimal design of VIS control should achieve a balance between response control and provisioning 


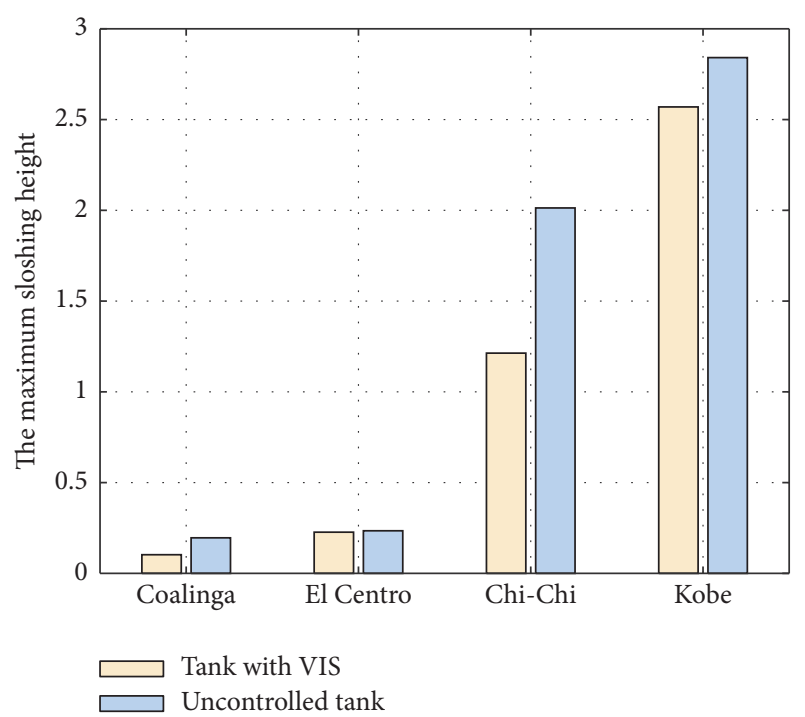

FIGURE 13: Maximum sloshing heights of the liquid in the tanks under different ground motions.

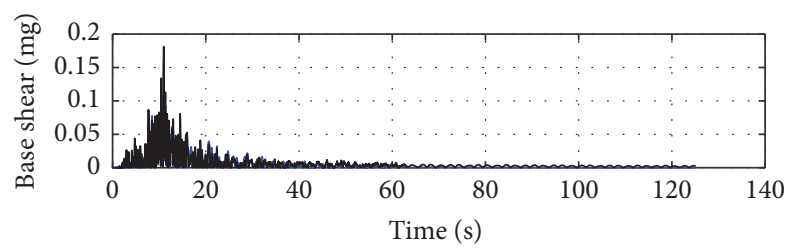

_ Tank with VIS

— Uncontrolled tank

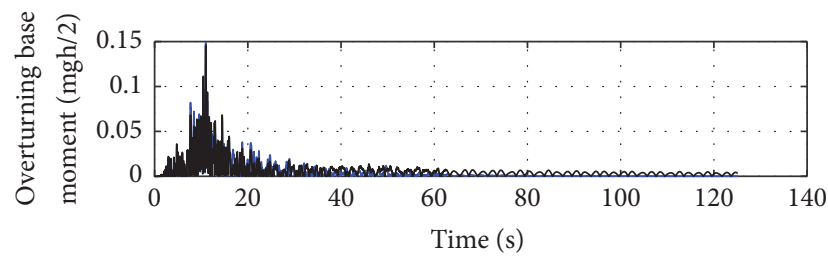

_ Tank with VIS

_ Uncontrolled tank

Figure 14: Base shears and overturning base moments of the tank with the VIS and the uncontrolled tank under the excitation of the Coalinga earthquake.

costs. The demand-based design approach proposed in this study can minimize costs and ensure that the response does not exceed a given limit.

(2) White noise excitation can be used to design the tank with the VIS under seismic excitation and simplify the calculation.

(3) The VIS is an effective structural control device for reducing the sloshing height responses of a tank system under dynamic excitation, such as white noise and earthquake excitations.
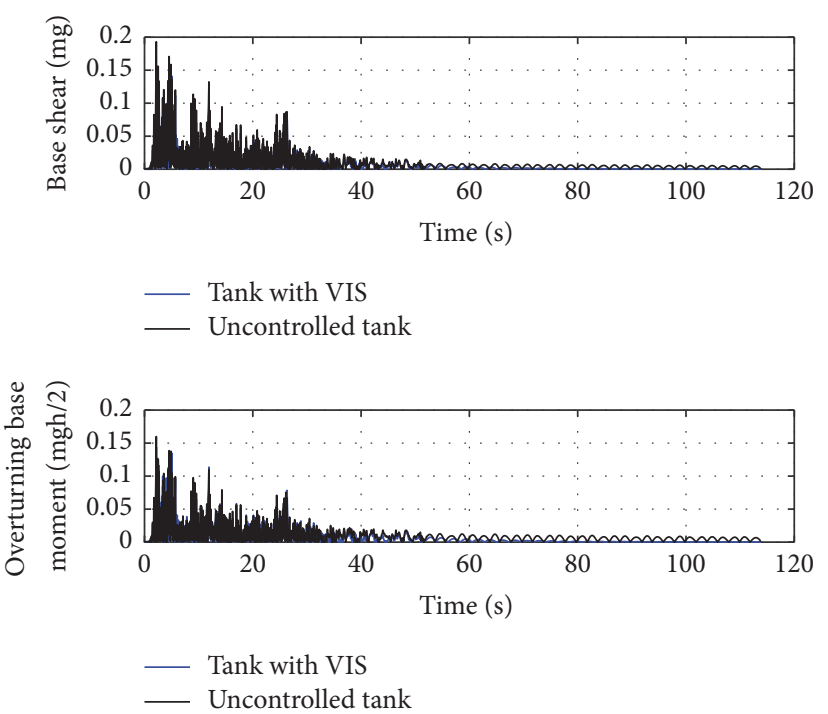

FIGURE 15: Base shears and overturning base moments of the tank with the VIS and the uncontrolled tank under the excitation of the El Centro earthquake.
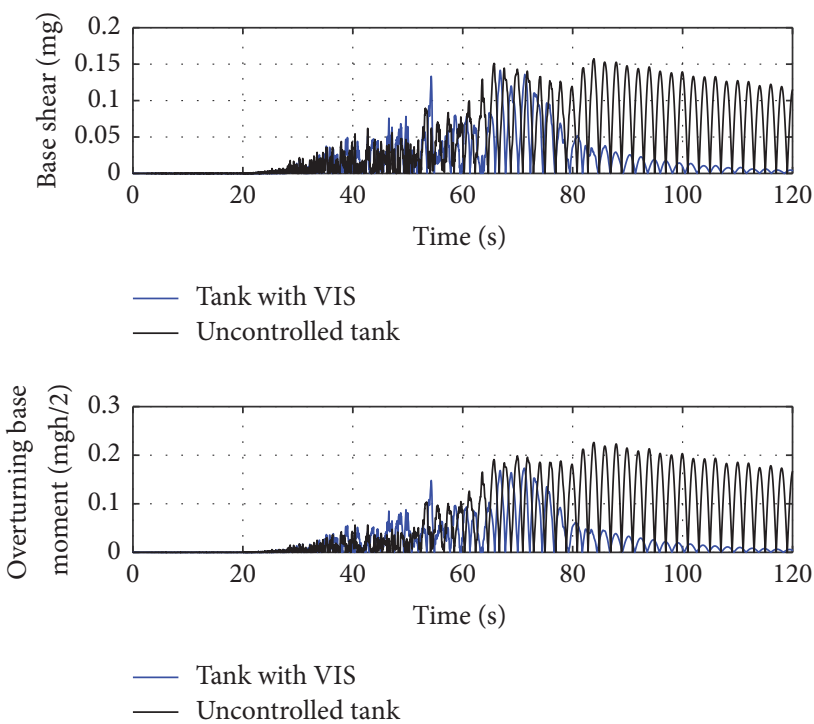

FIGURE 16: Base shears and overturning base moments of the tank with the VIS and the uncontrolled tank under excitation of the ChiChi earthquake.

(4) The parameter optimization design of the storage tank is based on the nonlinear optimization of a specific control objective in the feasible region of each parameter. Typically, the optimum design parameters for the tank are a combination of the upper limit of parameter $\mu_{c}$ and the other parameters. The storage tank with the VIS can be effectively controlled at a low cost to a considerable extent under the premise of small $\mu_{m}$ values. 

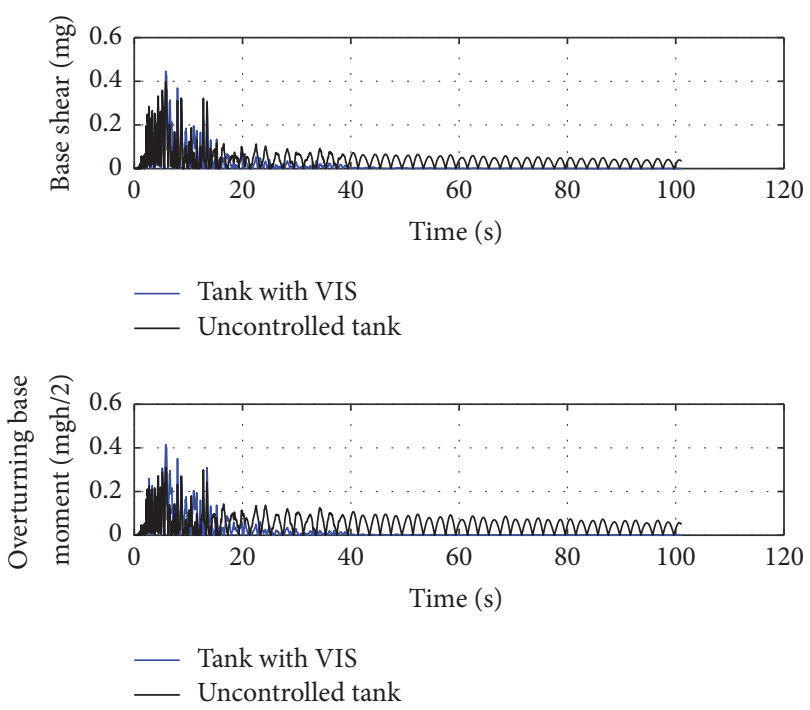

FIGURE 17: Base shears and overturning base moments of the tank with the VIS and the uncontrolled tank under the excitation of the Kobe earthquake.

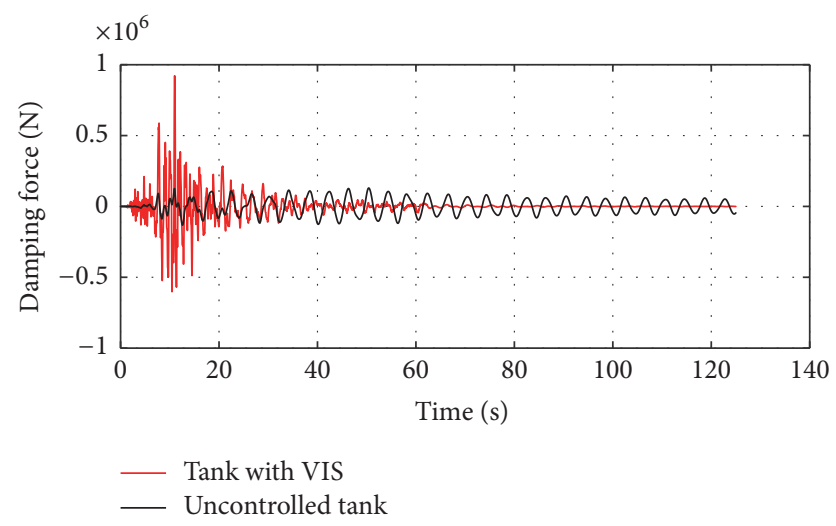

FIGURE 18: Output forces of the tank with the VIS and the uncontrolled tank under the excitation of the Coalinga earthquake.

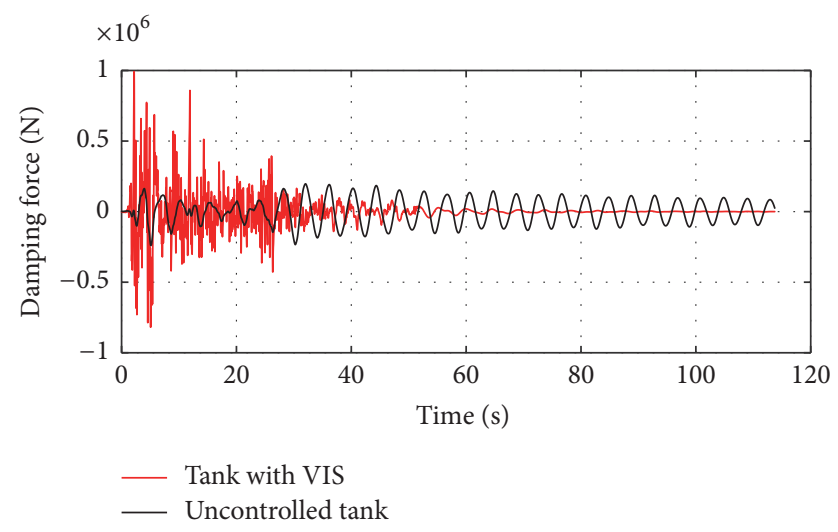

FIgURE 19: Output forces of the tank with the VIS and the uncontrolled tank under the excitation of the El Centro earthquake.

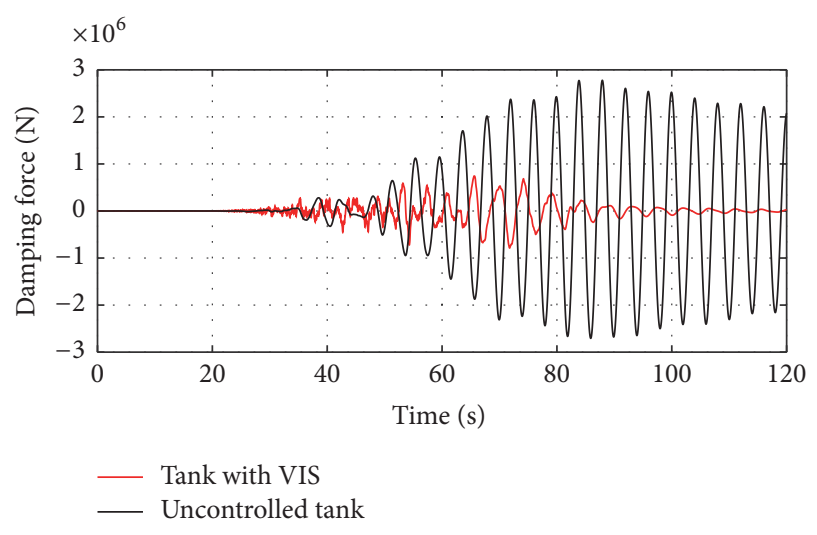

FIGURE 20: Output forces of the tank with the VIS and the uncontrolled tank under the excitation of the Chi-Chi earthquake.

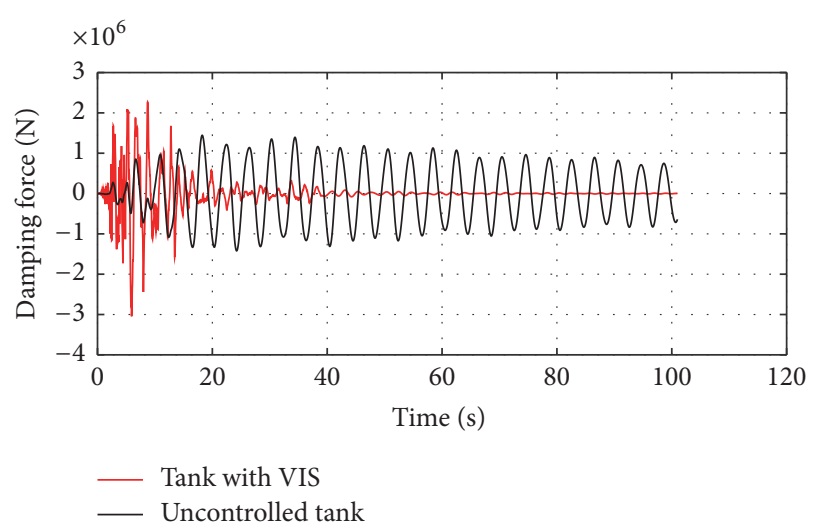

Figure 21: Output forces of the tank with the VIS and the uncontrolled tank under the excitation of the Kobe earthquake.

\section{Conflicts of Interest}

The authors declare that they have no conflicts of interest.

\section{Acknowledgments}

This study was supported by the Shanghai Pujiang Program under Grant no. 17PJ1409200, the National Natural Science Foundation of China under Grants nos. 51308418 and 51478356, and the Collaborative Research Project under International Joint Research Laboratory of Earthquake Engineering under Grant no. ILEE-IJRP-P1-P3-2016.

\section{References}

[1] M. K. Shrimali and R. S. Jangid, "Earthquake response of isolated elevated liquid storage steel tanks," Journal of Constructional Steel Research, vol. 59, no. 10, pp. 1267-1288, 2003.

[2] S. Zama, H. Nishi, M. Yamada, and K. Hatayama, "Damage of Oil Storage Tanks Caused by Liquid Sloshing in the 2003 Tokachi Oki Earthquake and Revision of Design Spectra in the Long-Period Range," in Proceedings of the 14 th World Conference on Earthquake Engineering, Beijing, China, 2008.

[3] K. Hatayama, "Lessons from the 2003 Tokachi-oki, Japan, earthquake for prediction of long-period strong ground motions and 
sloshing damage to oil storage tanks," Journal of Seismology, vol. 12 , no. 2, pp. 255-263, 2008.

[4] K. Hatayama, S. Zama, H. Nishi, M. Yamada, Y. Hirokawa, and R. Inoue, "Long-period strong ground motion and damage to oil storage tanks due to the 2003 Tokachi-oki Earthquake," Zisin Journal of the Seismological Society of Japan, vol. 57, no. 2, pp. 83-103, 2004.

[5] S. Yoshida, S. Zama, M. Yamada, K. Ishida, and T. Tahara, "Report on damage and failure of oil storage tanks due to the 1999 Chi-Chi Earthquake in Taiwan," in Proceedings of the ASME Pressure Vessels and Piping Conference, American Society of Mechanical Engineers, Atlanta, GA, USA, July 22-July 26, 2001.

[6] G. C. Manos and R. W. Clough, "Tank damage during the May 1983 Coalinga Earthquake," Earthquake Engineering \& Structural Dynamics, vol. 13, no. 4, pp. 449-466, 1985.

[7] K. H. Cho, M. K. Kim, Y. M. Lim, and S. Y. Cho, "Seismic response of base-isolated liquid storage tanks considering fluid-structure-soil interaction in time domain," Soil Dynamics and Earthquake Engineering, vol. 24, no. 11, pp. 839-852, 2004.

[8] K. Ikago, K. Saito, and N. Inoue, "Seismic control of singledegree-of-freedom structure using tuned viscous mass damper," Earthquake Engineering \& Structural Dynamics, vol. 41, no. 3, pp. 453-474, 2012.

[9] M. Ikenaga, Y. Fukumi, K. Ikago, and N. Inoue, "Application of friction damper with coupling mechanism designed in accordance with input ground motion levels to a base isolated detached house," Journal of Structural and Construction Engineering, vol. 78, no. 690, pp. 1413-1422, 2013.

[10] S. Zou, M. Ikenaga, N. Hori, K. Ikago, and N. Inoue, "Development of friction damper with coupling mechanism for displacement control of base-isolated system," AIJ Journal of Technology and Design, vol. 19, no. 43, pp. 855-860, 2013.

[11] E. Abali and E. Uçkan, "Parametric analysis of liquid storage tanks base isolated by curved surface sliding bearings," Soil Dynamics \& Earthquake Engineering, vol. 30, no. 1-2, pp. 21-31, 2010.

[12] M. K. Kim, M. L. Yun, S. Y. Cho, K. H. Cho, and W. L. Kang, "Seismic analysis of base-isolated liquid storage tanks using the BEFEBE coupling technique," Soil Dynamics Earthquake Engineering, vol. 22, no. 9-12, pp. 1151-1158, 2002.

[13] V. R. Panchal and R. S. Jangid, "Behaviour of liquid storage tanks with VCFPS under near-fault ground motions," Structure and Infrastructure Engineering, vol. 8, no. 1, pp. 71-88, 2012.

[14] M. R. Shekari, N. Khaji, and M. T. Ahmadi, "A coupled BEFE study for evaluation of seismically isolated cylindrical liquid storage tanks considering fluid-structure interaction," Journal of Fluids \& Structures, vol. 25, no. 3, pp. 567-585, 2009.

[15] M. K. Shrimali and R. S. Jangid, "Non-linear seismic response of base-isolated liquid storage tanks to bi-directional excitation," Nuclear Engineering and Design, vol. 217, no. 1-2, pp. 1-20, 2002.

[16] M. K. Shrimali and R. S. Jangid, "Seismic response of liquid storage tanks isolated by sliding bearings," Engineering Structures, vol. 24, no. 7, pp. 909-921, 2002.

[17] C. E. Ventura, W. D. Liam Finn, J.-F. Lord, and N. Fujita, "Dynamic characteristics of a base isolated building from ambient vibration measurements and low level earthquake shaking," Soil Dynamics and Earthquake Engineering, vol. 23, no. 4, pp. 313-322, 2003.

[18] R. Zhang, D. Weng, and X. Ren, "Seismic analysis of a LNG storage tank isolated by a multiple friction pendulum system,"
Earthquake Engineering and Engineering Vibration, vol. 10, no. 2, pp. 253-262, 2011.

[19] N.-S. Kim and D.-G. Lee, "Pseudodynamic test for evaluation of seismic performance of base-isolated liquid storage tanks," Engineering Structures, vol. 17, no. 3, pp. 198-208, 1995.

[20] F. Paolacci and R. Giannin, "Experimental investigation on the seismic behaviour of a base isolated steel liquid storage tank," in Proceedings of the 11th World Conference on Seismic Isolation, Energy Dissipation and Active Vibration Control of Struc, Guangzhou University, 2009.

[21] H. Luo, R. Zhang, and D. Weng, "Mitigation of liquid sloshing in storage tanks by using a hybrid control method," Soil Dynamics and Earthquake Engineering, vol. 90, pp. 183-195, 2016.

[22] A. Gedikli and M. E. Erguven, "Seismic analysis of a liquid storage tank with a baffle," Shock and Vibration Digest, vol. 32, no. 1, p. 29, 2000.

[23] A. Maleki and M. Ziyaeifar, "Sloshing damping in cylindrical liquid storage tanks with baffles," Journal of Sound and Vibration, vol. 311, no. 1-2, pp. 372-385, 2008.

[24] J. P. Den Hartog, Mechanical vibrations, Wiley, 1957.

[25] C. Pan, R. Zhang, H. Luo, C. Li, and H. Shen, "Demand-based optimal design of oscillator with parallel-layout viscous inerter damper," Structural Control and Health Monitoring, p. e2051.

[26] K. Kanai, "Semi-empirical formula for the seismic characteristics of the ground," Bulletin of Earthquake Research Institute, vol. 35, no. 2, pp. 309-325, 1957.

[27] G. W. Housner, "Dynamic pressures on accelerated fluid containers," Bulletin of the Seismological Society of America, vol. 47, no. 1, 1957.

[28] A.S. Veletsos, "Seismic response and design of liquid storage tanks," Guidelines for the seismic design of oil and gas pipeline systems, Technical Council on Lifeline Earthquake Engineering, ASCE, 255-370, pp. 443-461, 1984.

[29] J. H. Lin, W. Shen, and F. W. Williams, "Accurate high-speed computation of non-stationary random structural response," Engineering Structures, vol. 19, no. 7, pp. 586-593, 1997.

[30] C. Pan, R. Zhang, H. Luo, and H. Shen, "Target-based algorithm for baseline correction of inconsistent vibration signals," Journal of Vibration and Control, p. 107754631668901, 2017.

[31] C. Pan, R. Zhang, H. Luo, and H. Shen, "Baseline correction of vibration acceleration signals with inconsistent initial velocity and displacement," Advances in Mechanical Engineering, vol. 8, no. 10, pp. 1-11, 2016. 


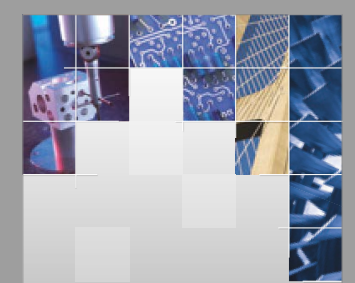

\section{Enfincering}
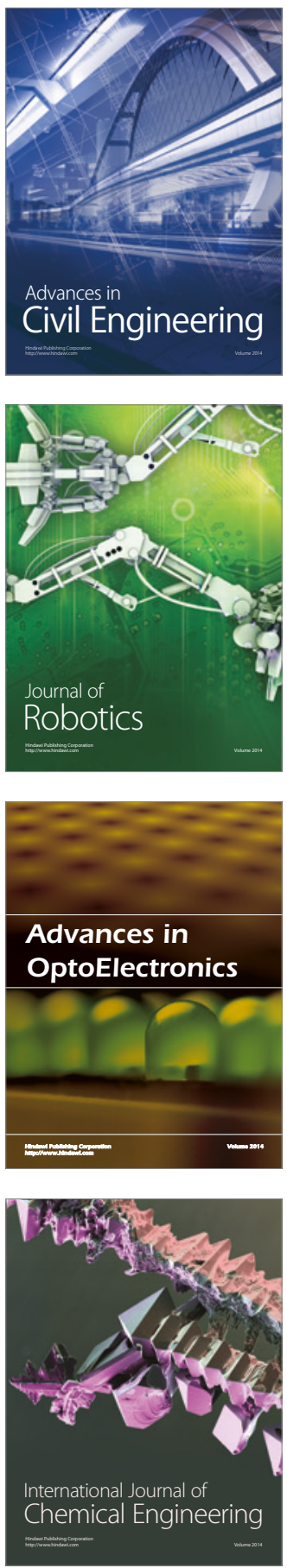

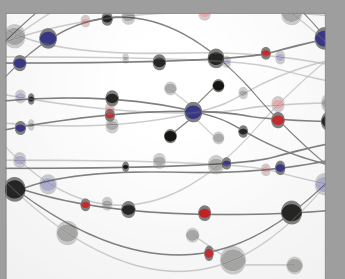

The Scientific World Journal

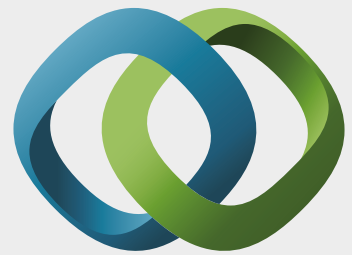

\section{Hindawi}

Submit your manuscripts at

https://www.hindawi.com
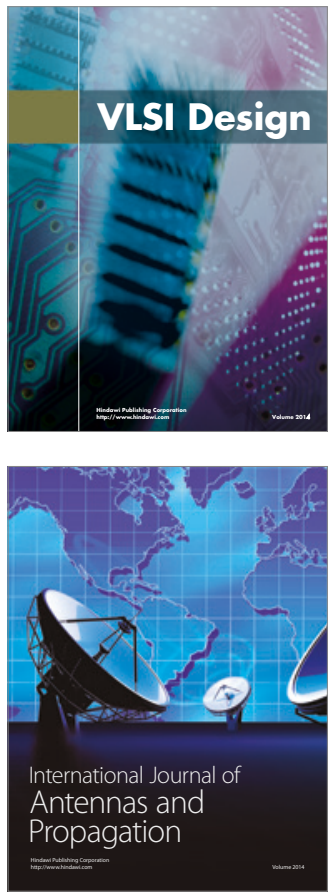

\section{Rotating}

Machinery
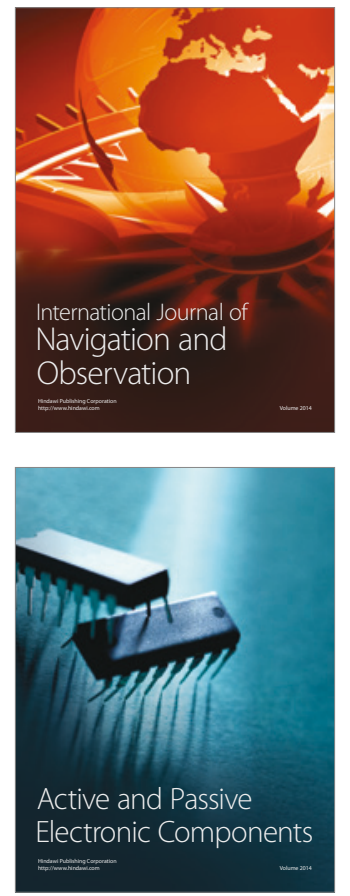
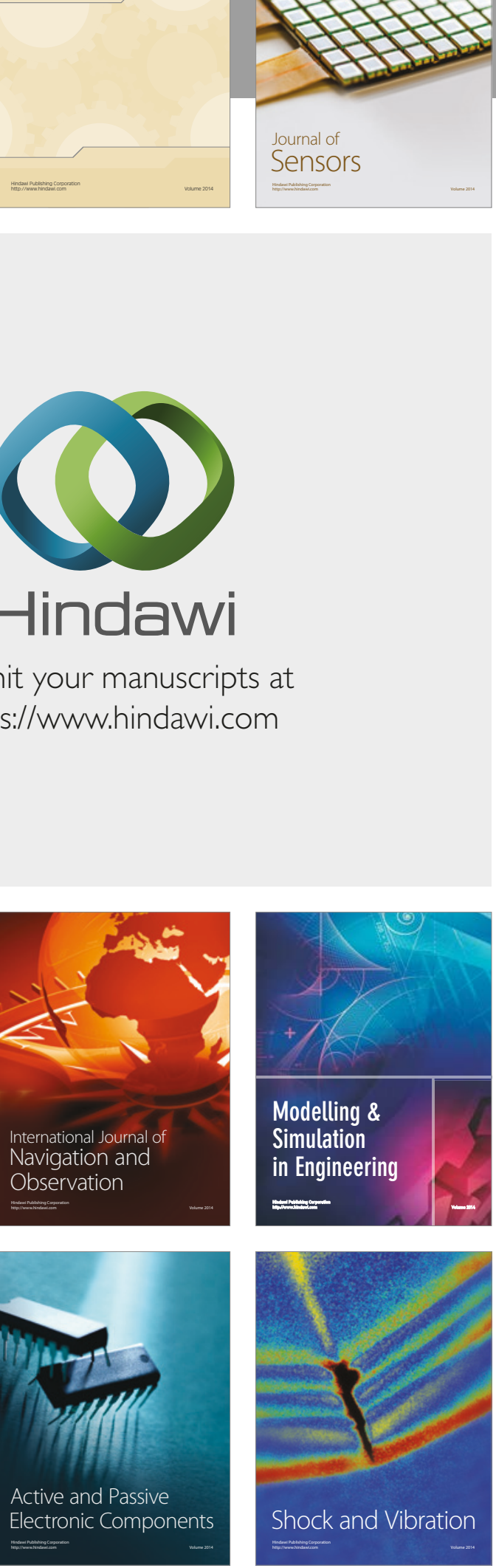
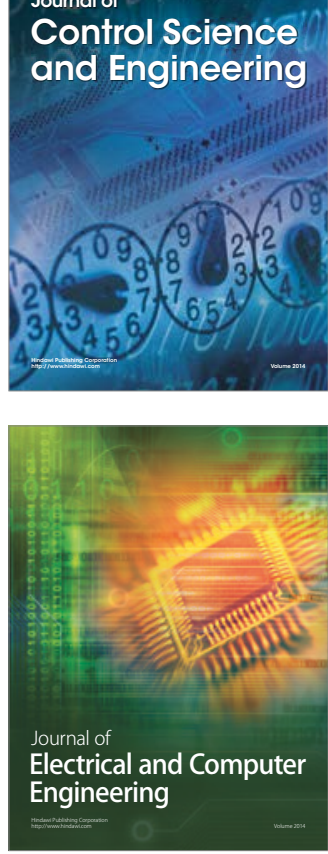

Distributed

Journal of

Control Science

and Engineering
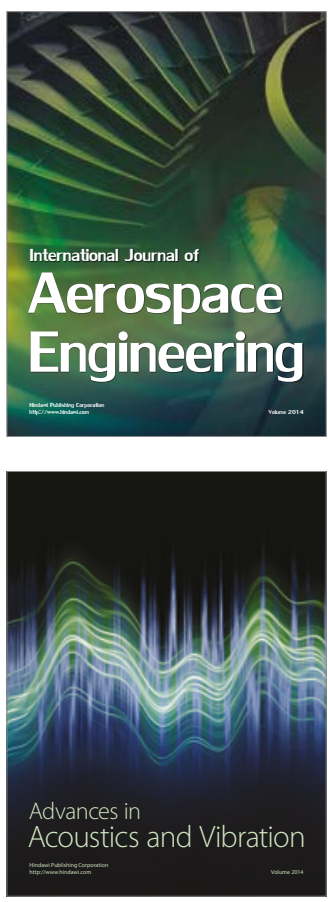

Sensor Networks 